\title{
Florística e estrutura da vegetação campestre nos Campos arbustivos de São Gabriel, Rio Grande do Sul, Brasil
}

\author{
Pedro Joel Silva da Silva Filho', Renato Backes Macedo², Mariana de Souza Vieira' \\ \& Paulo César Pereira das Neves ${ }^{3}$
}

\begin{abstract}
'Universidade Federal do Rio Grande do Sul, Programa de Pós-Graduação em Botânica, Laboratório de estudos em vegetação campestre. Av. Bento Gonçalves, 9500, prédio 43433, 91501-970, Porto Alegre, RS, Brasil. pedrojssf@yahoo.com.br

2Universidade Federal do Rio Grande do Sul, Programa de Pós-Graduação em Geociências, Laboratório de palinologia Marleni MarquesToigo. Av. Bento Gonçalves, 9500, prédio 43127, 91501-970, Porto Alegre, RS, Brasil.

${ }^{3}$ Universidade Luterana do Brasil, Laboratório de Geologia e Mineralogia. Av. Farroupilha 8001, Prédio I, 92420-280, Canoas, RS, Brasil.
\end{abstract}

Recebido em 20.1.2016

Aceito em 06.X.2017

DOI 10.21826/2446-8231201772305

RESUMO - Investigou-se a relação entre a distribuição das espécies e as condições abióticas locais de um campo natural no município de São Gabriel. Quatro fitofisionomias foram definidas a priori (campo rupestre, campo seco, campo úmido e campo brejoso) e avaliadas se as mesmas caracterizam diferentes comunidades locais. O levantamento quantitativo foi realizado através de 40 unidades amostrais de $1 \mathrm{~m}^{2}$ cada. A lista florística contém 244 espécies, das quais 199 foram amostradas na análise fitossociológica. As três primeiras espécies com o maior índice de valor de importância foram distintas em cada fitofisionomia. Houve maior similaridade entre o campo rupestre e o campo seco, os quais também apresentaram o maior valor de diversidade. A análise exploratória multivariada revelou um agrupamento das UAs de mesma fitofisionomia, comprovando que que as fitofisionomias observadas constituem comunidades heterogêneas.

Palavras-chave: Campos, fitossociologia, pampa, Serra do Sudeste

\begin{abstract}
Floristic and structure of grassland vegetation in the Campos Arbustivos of São Gabriel (Arroio do Salso headwaters), Rio Grande do Sul, Brazil. In this study we investigate the relationship between the distribution of species and local abiotic conditions of a natural grassland in São Gabriel municipality. Four phytophysiognomies were defined a priori (rocky grassland, dry grassland, wet grassland and marshy grasslands) and tested whether they could characterize different local communities. The quantitative survey employed 40 sampling units of $1 \mathrm{~m}^{2}$. The floristic list contains 244 species, of which 199 were sampled in the phytosociological analysis. The first three species with the highest importance value index were different in each phytophysiognomy. There is higher similarity among the rocky grassland and dry grassland, which also showed the highest diversity values. Multivariate exploratory analysis grouped sample units by it's phytophysiognomy, evidencing that the phytophysiognomies observed perform heterogeneous communities.
\end{abstract}

Keywords: Campos, pampa, phytosociology, Serra do Sudeste

\section{INTRODUÇÃO}

Os biomas campestres atuais, em escala global, abrangem uma área aproximada de 39 milhões de $\mathrm{km}^{2}$, o que corresponde a uma quarta parte das áreas emersas (Constanza et al. 1997). Os Pastizales do Río de la Plata (PRP) constituem um dos ecossistemas campestres mais extensos das regiões temperadas, cobrindo cerca de 750.000 $\mathrm{km}^{2}$ (Bilenca \& Miñarro 2004). Localiza-se no Sudeste da América do Sul, entre as latitudes $28^{\circ}$ e $38^{\circ} \mathrm{Sul}$ (Soriano 1991) (Fig. 1A). A partir de Burkart (1975) e Soriano (1991) os PRP têm sido caracterizados em duas subdivivisões regionais: Pampas, nas planícies centro-leste da Argentina; e Campos, no nordeste da Argentina, Uruguai e Sul do Brasil. Estes fornecem essenciais serviços ecossistêmicos como o armazenamento de carbono, a regulação dos ciclos hidrológicos, a manutenção dos polinizadores, o controle de erosão e a produção de forragem para a atividade de pecuária (Boldrini et al. 2010, Overbeck et al. 2013, 2015, Andrade et al. 2015, Pillar et al. 2015, Modernel et al. 2016), entre outros (Gibson 2009).

No território brasileiro, os Campos se restringem ao Rio Grande do Sul, com uma cobertura de aproximadamente $176.496 \mathrm{~km}^{2}$, correspondendo em torno de $63 \%$ da área total deste Estado (IBGE 2004). No entanto, de acordo com o atual sistema de classificação dos biomas brasileiros, os Campos são denominados como "Pampa" (IBGE 2004), terminologia ainda controversa e contestada por autores como Ab'Sáber (2005) e Overbeck et al. (2007). Portanto, neste artigo optou-se pela nomenclatura sensu Burkart (1975) e Soriano (1991), por haver conformidade com a literatura internacional.

Apesar da aparência topograficamente uniforme, os PRP abrigam uma elevada riqueza de plantas vasculares, ainda não totalmente conhecidas, cuja conservação historicamente tem sido negligenciada (Bilenca \& Miñarro, 


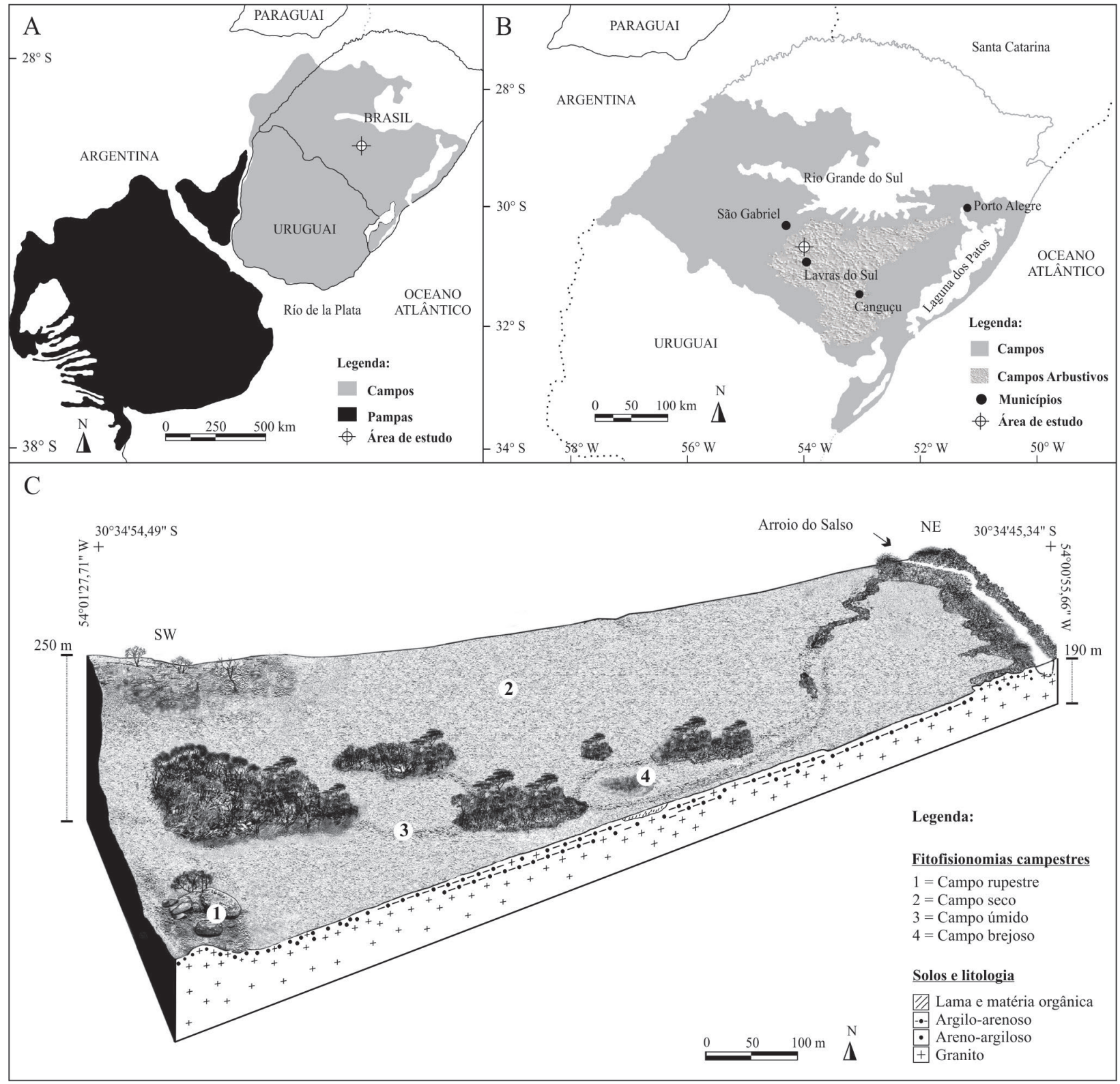

Figs. 1A-C. Localização da área de estudo. A. Abrangência dos PRP, dividida em subdivisões regionais: Pampas e Campos (Soriano 1991), adaptado de Tonello \& Prieto (2008) e Mourelle (2011); B. Distribuição dos Campos arbustivos no Rio Grande do Sul, modificado de Hasenack et al. (2010); C. Ilustração mostrando os aspectos fisionômicos nas cabeceiras do Arroio do Salso, em São Gabriel.

2004, Overbeck et al. 2007, 2013, 2015). Somente nos Campos do Rio Grande do Sul, onde restam 35,84\% de sua cobertura original (CSR/IBAMA 2011), estimativas conservadoras indicam a ocorrência de aproximadamente 2.300 espécies campestres (Ilsi Iob Boldrini, comunicação pessoal, 2015).

Recentemente, Hasenack et al. (2010) com base nas informações da flora campestre e do meio abiótico, delimitaram múltiplos sistemas ecológicos para os Campos (ou Savanas uruguaias), incluindo os Campos arbustivos aqui estudados. No Rio Grande Sul, os Campos arbustivos totalizam uma área em torno de $34.124 \mathrm{~km}^{2}$ sobre as rochas neoproterozoicas do Escudo Sul-rio-grandense (Heinrich Hasenack, comunicação pessoal, 2016), figura 1B. A fisionomia campestre nesse sistema ecológico é principalmente resultante do equilíbrio entre diversas espécies das famílias Poaceae e Asteraceae, guardando semelhanças com o uruguaio, em continuidade ao sul (Hasenack et al. 2010). Ademais, cabe ressaltar a ampla representação das famílias Cyperaceae e Rubiaceae, além da grande quantidade de espécies forrageiras, especialmente da Fabaceae (Boldrini 2009). Também ocorrem espécies endêmicas em diferentes ambientes como às cactáceas, associadas à vegetação rupestre (Boldrini et al. 2010).

De modo geral, quando comparado aos demais sistemas ecológicos sul-rio-grandenses, a matriz campestre nos Campos arbustivos se apresenta relativamente melhor preservada (Cordeiro \& Hasenack 2009; Andrade et al. 2015). Isso se deve, sobretudo, à acentuada presença de afloramentos graníticos e de solos rasos (litossolos) 
na região, o que dificulta a expansão da agricultura. Historicamente estas áreas eram utilizadas exclusivamente para atividades pastoris. Porém, na última década, as pastagens nativas vêm sendo amplamente convertidas em culturas de Eucalyptus spp., Glycine spp. e Triticum spp., entre outras, comprometendo a manutenção da biodiversidade e do ambiente como um todo.

Apesar dos avanços recentes do conhecimento florístico e fitossociológico da vegetação campestre nos Campos arbustivos do Rio Grande do Sul, as publicações concentram-se nos morros graníticos de Porto Alegre (Morro da Polícia: Boldrini et al. 1998; Morro Santana: Overbeck et al. 2006; Morro do Osso: Ferreira et al. 2010; Morro São Pedro: Setubal \& Boldrini 2010, 2012, Jardim Botânico de Porto Alegre: Dresseno \& Overbeck 2013, Rolim et al. 2014), que apesar de na classificação de Hasenack (2010) não compreender este sistema ecológico, possuem características geológicas e botânicas similares. Em áreas do interior do estado há poucos trabalhos no tema, realizados nos municípios de Canguçu (Caporal \& Boldrini 2007) e Lavras do Sul (Zocche 2002, Sippel 2003, Frizzo \& Porto 2004), as três últimas referências restritas à localidade da Mina Volta Grande, demonstrando assim, que uma extensa área neste sistema ecológico ainda é pouco conhecida. Especificamente nos Campos Arbustivos de São Gabriel, que abrangem a porção sul e sudeste deste município, as descrições da flora campestre dão-se de forma sucinta nas obras bastante genéricas (Rambo 1956, Müller 1962, Lindman \& Ferri 1974, Porto 2002).

Assim, este estudo teve por objetivo caracterizar a composição florística e a estrutura da vegetação campestre em uma área conservada nos Campos arbustivos de São Gabriel, investigando a relação entre a distribuição das espécies e as condições abióticas locais.

\section{MATERIAL E MÉTODOS}

\section{Localização e aspectos fisiográficos da área de estudo}

A área em estudo situa-se no município de São Gabriel (cabeceiras do Arroio do Salso, distrito do Cerro do Ouro), Rio Grande do Sul, Brasil (Fig. 1B). O acesso se dá por estradas vicinais nas propriedades rurais: Salso $\left(30^{\circ} 34^{\prime} 54,49^{\prime \prime} \mathrm{S}\right.$; $\left.54^{\circ} 01^{\prime} 27,71^{\prime \prime} \mathrm{W}\right)$ e Horizontina $\left(30^{\circ} 34^{\prime} 45,34^{\prime \prime} \mathrm{S}\right.$; $54^{\circ} 00^{\prime} 55,66^{\prime}$ 'W), a partir da rodovia estadual RSC-473, entre as cidades de São Gabriel e Lavras do Sul.

Em termos geológicos, a área situa-se nos terrenos neoproterozoicos do Escudo Sul-rio-grandense (Serra do Sudeste; Domínio São Gabriel), na porção meridional da Província Mantiqueira (CPRM 2006). Situa-se no Complexo Cambaí e Suíte da Meia Lua (indivisos), com idades entre 740-680 milhões de anos (Vedana \& Philipp 2016). Localmente, as litologias correspondem ao Granito São Manoel, além da presença subordinada de rochas metamórficas de natureza xistosa, na maioria das vezes não aflorantes (CPRM 2006, Vedana \& Philipp 2016).

A geomorfologia é caracterizada por um conjunto de relevos ondulados, com topos de forma convexa e altitudes variáveis entre 200 a 300 m, esculpidas no Granito São Manoel. Trata-se de uma zona de cabeceiras de primeira ordem; as encostas do terreno são entalhadas comumente por sulcos, em geral pouco profundos, às vezes, denotando um controle estrutural e apresentando um grau de instabilidade morfodinâmica muito forte (IBGE 2003).

Quanto à pedologia predominam luvissolos crômicos órticos típicos, da Unidade Cambaí (Streck et al. 2008), caracterizados pelo acúmulo subsuperficial de argila e presença esparsa de afloramentos rochosos. A origem destes luvissolos corresponde às rochas xistosas existentes na região, cuja alteração formou solos autóctones marcados por horizontes pouco profundos, bem drenados, ligeiramente ácidos e providos de boa reserva de nutrientes para as plantas (Reinert et al. 2007, Goffermann et al. 2015).

O clima, conforme a classificação de Köppen enquadrase no tipo Cfa, descrito como uma zona temperada quente e úmida, com as quatro estações do ano bem definidas, ausência de estação seca e precipitações pluviométricas distribuídas regularmente ao longo do ano (Peel et al. 2007, Alvares et al. 2013). Especificamente, no município de São Gabriel, as médias das temperaturas mínimas e máximas anuais são respectivamente $14,2^{\circ} \mathrm{C}$ e $24,9^{\circ} \mathrm{C}$ e a média de precipitação pluviométrica acumulada anual é 1.590,5 $\mathrm{mm}$ (Wrege et al. 2011).

Com relação à cobertura vegetal, a matriz campestre mostra-se heterogênea e bem preservada, com diferentes fitofisionomias distinguíveis em campo e definidas a priori como: campo rupestre, campo seco, campo úmido e campo brejoso (Fig. 1C).

O campo rupestre (Fig. 2A) situa-se nos setores mais elevados da área e constitui-se de afloramentos graníticos e litossolos provenientes dos mesmos, com a presença de uma vegetação xerofítica, representada predominantemente por poáceas cespitosas e cactáceas. O campo seco (Fig. 2B) ocorre ao longo das encostas convexas e seu substrato é tipicamente areno-argiloso, com uma vegetação em dois estratos distintos, o inferior representado principalmente por poáceas estoloníferas e rizomatosas, e o superior por asteráceas subarbustivas. O campo úmido (Fig. 2C) ocorre ao longo das encostas côncavas, num limite difuso com o campo seco, sendo mais expressivo no sopé das mesmas, em porções relativamente mais planas, constituindo-se basicamente por sedimentos argilo-arenosos onde há maior retenção de água e domínio de uma vegetação herbácea caracterizada por poáceas e ciperáceas estoloníferas e rizomatosas. O campo brejoso (Fig. 2D) refere-se a uma pequena bacia de captação de água e sedimentos provenientes das vertentes à montante, preenchida por lama e matéria orgânica, com uma vegetação tipicamente higrófila.

\section{Florística}

A vegetação campestre foi inventariada durante a primavera de 2013 e 2014 pelo método do caminhamento 

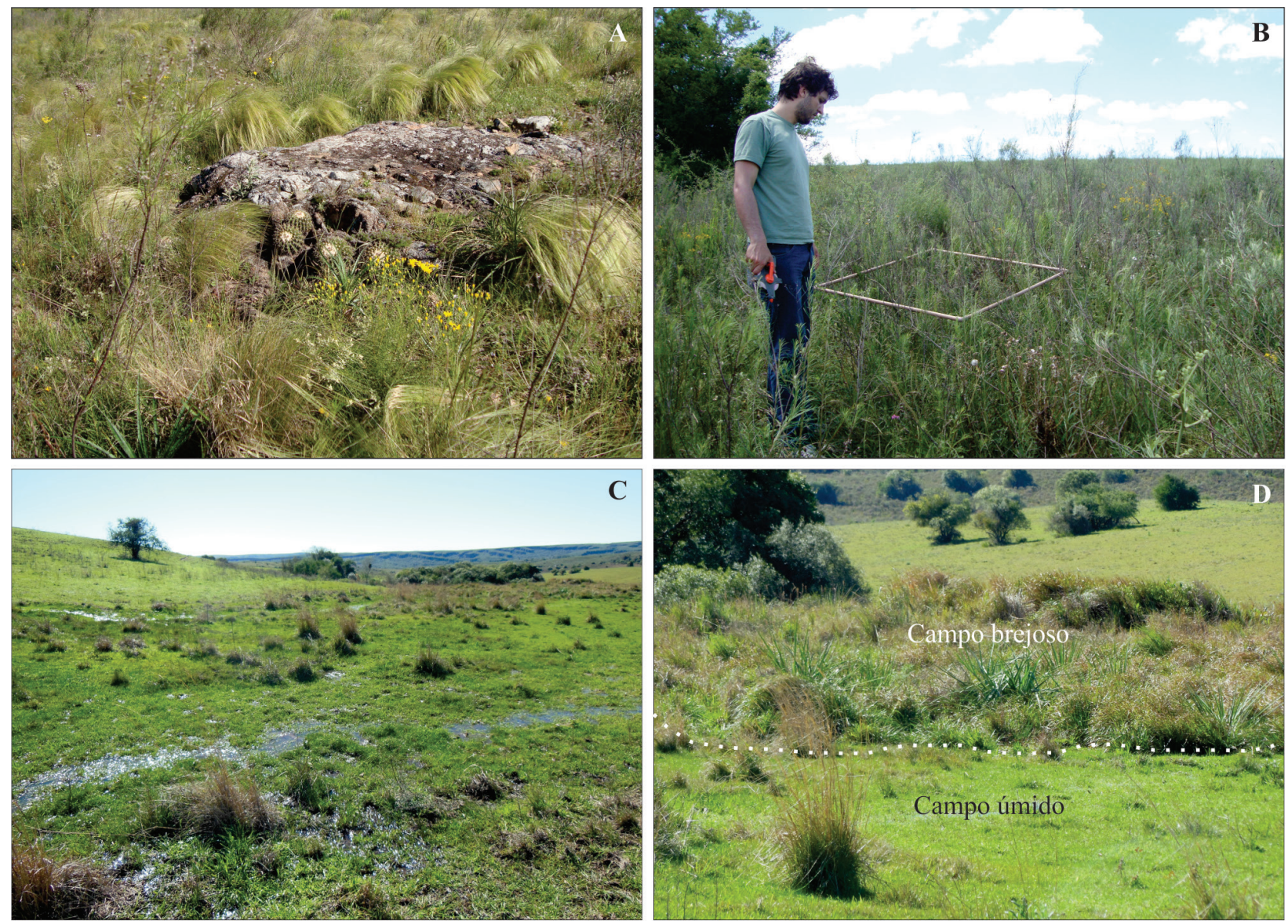

Figs. 2A-D. Fitofisionomias campestres nas cabeceiras do Arroio do Salso. A. Campo rupestre; B. Campo seco; C. Campo úmido; D. Campo brejoso.

(Filgueiras et al. 1994). A identificação dos táxons foi realizada diretamente em campo e, quando não foi possível, coletou-se material para exsicatas e posterior determinação por especialistas. A nomenclatura taxonômica foi consultada na base de dados IPNI (2015) e Flora do Brasil (2020), que seguem a classificação do Angiosperm Phylogeny Group (APG III 2009). Adicionalmente, verificou-se a ocorrência de espécies ameaçadas de extinção, conforme a lista constante no Decreto Estadual n ${ }^{\circ} .52 .109 / 2014$.

\section{Levantamento quantitativo}

O levantamento quantitativo foi realizado utilizando-se o método amostral de superfície, em unidades amostrais (UAs) de $1 \mathrm{~m}^{2}$ cada. Foram levantadas 10 UAs de forma aleatória em cada fitofisionomia. Em cada UA foi estimada a cobertura de todas as espécies (exceto briófitas), usandose a escala de Braun-Blanquet (1979), modificada para os seguintes intervalos: " 0,1 " = cobertura até $1 \%$; " 0,5 " $=$ cobertura entre 1,1 e $5 \%$; " $1 "$ = cobertura entre 5,1 e $10 \%$; "2"= cobertura entre 10,1 e $20 \%$; " 3 " = cobertura entre 20,1 e $30 \%$; " 4 " = cobertura entre 30,1 e $40 \%$; " 5 " $=$ cobertura entre 40,1 e $50 \%$; " 6 " = cobertura entre 50,1 e $60 \%$; "7" = cobertura entre 60,1 e $70 \%$; " 8 " = cobertura entre 70,1 e $80 \%$; "9" = cobertura entre 80,1 e $90 \%$; " 10 " $=$ cobertura entre 90,1 e $100 \%$.

\section{Procedimento analítico}

Para cada espécie encontrada nas UAs foram calculados os seguintes parâmetros: frequência absoluta (FA) e relativa (FR), cobertura absoluta (CA) e relativa (CR) e o índice de valor de importância (IVI) (MüellerDombois \& Ellenberg 1974). Foram também avaliados a riqueza, o índice de similaridade de Jaccard (J) e a diversidade específica por meio do índice de ShannonWiener ( $\left.\mathrm{H}^{\prime}\right)$, com base nos dados de presença e ausência para cada uma das áreas.

Para avaliar se as fitofisionomias campestres definidas a priori compõem distintas comunidades locais, foi realizada uma análise de Ordenação por Coordenadas Principais (PCoA), a partir de uma matriz de dados contendo todas as espécies registradas no censo quantitativo da vegetação e seus respectivos valores de CR em cada UA. Tais valores de CR foram previamente transformados em raiz quadrada para reduzir a variância dos táxons dominantes. Utilizouse como medida de semelhança a distância de cordas, comparando as UAs. Os táxons com os maiores coeficientes de correlação $(0,5$ e $-0,5)$ em relação aos dois primeiros eixos da ordenação foram plotados em um diagrama. A significância dos eixos foi avaliada pela técnica de bootstrap com 1.000 interações (Pillar 1999). Estes cálculos foram efetuados no software MULTIV (Pillar 2006). 


\section{RESULTADOS E DISCUSSÃO}

Uma das dificuldades encontradas para discussão deste trabalho foi encontrar outros estudos fitossociológicos em áreas adjacentes, limitando assim o aspecto comparativo. Este artigo é pioneiro na temática para os campos naturais desta região, uma vez que os trabalhos realizados em áreas lindeiras, no município de Lavras do Sul, foram desenvolvidos na Mina Volta Grande, com ambiente antropizado. Outra dificuldade refere-se aos diferentes métodos utilizados nos levantamentos quantitativos da vegetação campestre. O único trabalho similar existente nos Campos arbustivos do Rio Grande do Sul é o de Setubal \& Boldrini (2012), efetivado no Morro São Pedro, em Porto Alegre. No entanto, não é segura uma comparação entre áreas tão distantes, muito embora a flora e a geologia sejam relativamente similares. Ademais, os campos do Morro São Pedro se encontram numa área de contato com elementos do bioma da Mata Atlântica (IBGE 2004) e condicionantes de maior umidade pela regulação moderadora do Oceano Atlântico e do sistema Guaíba.

\section{Riqueza de espécies}

No inventário florístico foram reconhecidos 244 táxons, pertencentes a 51 famílias e 152 gêneros (Quadro 1). Deste total, 222 são herbáceas, 21 subarbustivas e uma arbustiva. As quatro famílias com maior riqueza foram Poaceae (54 spp.), Asteraceae (48 spp.), Cyperaceae (23 spp.) e Fabaceae (11 spp.), acompanhadas por Rubiaceae (oito spp.), Caryophyllaceae, Iridaceae, Oxalidaceae (seis spp. cada) e Verbenaceae (cinco spp.).

$\mathrm{O}$ elevado número de espécies relacionadas à flora campestre na área levantada (cerca de $10 \mathrm{ha}$ ), considerandose as quatro fitofisionomias, corrobora a riqueza deste sistema ecológico. Estes dados correspondem em torno de $10,6 \%$ da flora campestre para ecorregião dos Campos do Rio Grande do Sul, estimada em 2.300 spp. (Ilsi Iob Boldrini, comunicação pessoal, 2015). Outro aspecto que ressalta a conservação da matriz campestre local é a baixa representatividade de espécies naturalizadas (dez), exóticas (uma) e, a ausência de invasoras (Flora do Brasil 2020). Estudos prévios realizados em áreas adjacentes, na Mina Volta Grande, no município de Lavras do Sul (Zocche 2002, Sippel 2003, Frizzo \& Porto 2004), apresentam dados florísticos apenas baseados em levantamentos fitossociológicos, que são discutidos a seguir.

Dentre as quatro fitofisionomias definidas, o campo rupestre e o campo seco, além de maior riqueza, denotam também um maior número de espécies exclusivas com respectivamente $50 \mathrm{spp}$. e $48 \mathrm{spp}$. (Quadro 1). Por outro lado, analisando-se o número total de espécies levantadas no campo brejoso (60 spp.) e o número de táxons com ocorrência restrita neste ambiente (33 spp.), a porcentagem de plantas exclusivas (55\%) é superior às demais tipologias campestres. Possivelmente, isto se deva às características particulares deste ambiente, especialmente pela saturação de água e solos ligeiramente ácidos no campo brejoso, o que vai de encontro aos estudos de Reinert et al. (2007) e Goffermann et al. (2015) para a região.

Segundo a lista da flora ameaçada de extinção do Rio Grande do Sul, verificou-se a existência de nove espécies constantes neste inventário florístico, citadas em diferentes categorias: Cypella pusilla Benth. \& Hook.f., como criticamente em perigo; Frailea buenekeri subsp. densispina Hofacker \& Herm, Gymnocalycium denudatum (Link \& Otto) Pfeiff. ex Mittler, Parodia erinacea (Haw.) N. P. Taylor e Porophyllum linifolium (L.) DC., em perigo; Parodia mammulosa Trin., Parodia ottonis (Lehmann) N. P. Taylor e Kelissa brasiliensis (Baker) Ravenna, como vulnerável; Pfaffia gnaphaloides (L. f.) Mart., como quase ameaçada (Figs. 3A-I).

\section{Fitossociologia}

No levantamento quantitativo da vegetação campestre foram identificados 199 táxons, distribuídos em 42 famílias e 125 gêneros (Quadro 1). No campo brejoso foi considerada uma espécie de monilófita (Thelypteris sp.), as demais, foram todas angiospermas. As cinco famílias que apresentaram maior riqueza foram: Poaceae (51 spp.), Asteraceae (39 spp.), Cyperaceae (19 spp.), Fabaceae (nove spp.) e Rubiaceae (sete spp.). Avaliando a soma do número de espécies das famílias supracitadas, as mesmas totalizam $63 \%$ dos táxons registrados nas UAs, sendo que as três primeiras famílias correspondem a $55 \%$ desse total.

A riqueza específica evidenciada neste censo quantitativo foi muito superior ao registrado na Mina Volta Grande, em Lavras do Sul, e numericamente similar às registradas nos morros graníticos de Porto Alegre e no município de Canguçu (Tab. 1).

Ainda, com base nos parâmetros quantitativos, observouse que as três primeiras espécies com maior IVI, foram distintas em cada fitofisionomia (Tabs. 2A-D). No campo rupestre e no campo seco, os táxons mais representativos pertencem às famílias Poaceae e Asteraceae, enquanto no campo úmido e no campo brejoso há uma substituição da família Asteraceae por Cyperaceae. Tal fato também é notável no gráfico acumulativo do somatório dos IVI por família, onde foram consideradas todas as espécies levantadas (Figs. 4A-D).

No campo rupestre, com maior IVI, sobressaíram: Aristida jubata, A. venustula e Baccharis crispa (Tab. 2A). Poaceae às demais famílias, possui elevado IVI devido à ampla CR das espécies que ali ocorrem, cujos espécimes formam grande touceiras. No campo seco, destacaramse: Paspalum notatum, Vernonanthura nudiflora e Vulpia bromoides (Tab. 2B). Outra espécie com elevado IVI nestas fitofisionomias corresponde a Eryngium horridum, cujos valores de CR mostraram-se superiores as suas FR. Examinado o somatório dos IVI das espécies de Poaceae e Asteraceae, verificou-se que as mesmas totalizaram mais de $60 \%$ nos campos rupestre e seco (Figs. 4A, B).

No campo úmido, com maior IVI, predominaram: Axonopus affinis, Paspalum pumilum, Eleocharis bonariensis e E. viridans, seguidas de Luziola peruviana, 
Quadro 1. Inventário florístico com informações de hábitat $(\mathrm{CRv}=$ campo rupestre; $\mathrm{CSv}=$ campo seco; $\mathrm{CUv}=$ campo úmido; $\mathrm{CBv}=$ campo brejoso), origem e hábito. A sigla "LQ" indica as espécies registradas no levantamento quantitativo.

\begin{tabular}{|c|c|c|c|c|c|c|c|}
\hline Famílias/Espécies & $\mathrm{CRv}$ & $\mathrm{CSv}$ & CUv & $\mathrm{CBv}$ & Origem & Hábito & LQ \\
\hline \multicolumn{8}{|l|}{ Angiospermas } \\
\hline \multicolumn{8}{|l|}{ Acanthaceae } \\
\hline Hygrophila costata Nees \& T. Nees & - & - & - & $\mathrm{x}$ & nativa & herbáceo & $\mathrm{x}$ \\
\hline \multicolumn{8}{|l|}{ Alismataceae } \\
\hline Echinodorus grandiflorus (Cham. \& Schltdl.) Micheli & - & - & - & $\mathrm{x}$ & nativa & herbáceo & $\mathrm{x}$ \\
\hline \multicolumn{8}{|l|}{ Alliaceae } \\
\hline Nothoscordum cf. bivalve (L.) Britton & $\mathrm{x}$ & $\mathrm{x}$ & - & - & nativa & herbáceo & $\mathrm{x}$ \\
\hline \multicolumn{8}{|l|}{ Amaranthaceae } \\
\hline Chenopodium retusum (Moq.) Juss. ex Moq. & $\mathrm{x}$ & - & - & - & nativa & herbáceo & $\mathrm{x}$ \\
\hline Pfaffia gnaphaloides (L. f.) Mart. & $\mathrm{x}$ & - & - & - & nativa & herbáceo & $\mathrm{x}$ \\
\hline P. tuberosa (Spreng.) Hicken & $\mathrm{x}$ & $\mathrm{x}$ & - & - & nativa & herbáceo & $\mathrm{x}$ \\
\hline \multicolumn{8}{|l|}{ Apiaceae } \\
\hline Cyclospermum leptophyllum (Pers.) Britton \& P.Wilson & $\mathrm{x}$ & $\mathrm{x}$ & $\mathrm{x}$ & - & nativa & herbáceo & $\mathrm{x}$ \\
\hline Eryngium horridum Malme & $\mathrm{x}$ & $\mathrm{x}$ & - & - & nativa & herbáceo & $\mathrm{x}$ \\
\hline E. nudicaule Lam. & $\mathrm{x}$ & $\mathrm{x}$ & - & - & nativa & herbáceo & $\mathrm{x}$ \\
\hline E. pandanifolium Cham. \& Schltdl. & - & - & - & $\mathrm{x}$ & nativa & herbáceo & $\mathrm{x}$ \\
\hline Lilaeopsis brasiliensis (Glaz.) Affolter & - & - & $\mathrm{x}$ & $\mathrm{x}$ & nativa & herbáceo & $\mathrm{x}$ \\
\hline \multicolumn{8}{|l|}{ Araliaceae } \\
\hline Hydrocotyle exigua Malme & - & $\mathrm{x}$ & - & - & nativa & herbáceo & $\mathrm{x}$ \\
\hline H. bonariensis Lam. & - & - & $\mathrm{x}$ & - & nativa & herbáceo & $\mathrm{x}$ \\
\hline H. ranunculoides L.f. & - & - & $\mathrm{x}$ & $\mathrm{x}$ & nativa & herbáceo & $\mathrm{x}$ \\
\hline \multicolumn{8}{|l|}{ Asclepiadaceae } \\
\hline Oxypetalum solanoides Hook. \& Arn. & - & $\mathrm{x}$ & - & - & nativa & herbáceo & - \\
\hline \multicolumn{8}{|l|}{ Asteraceae } \\
\hline Acanthostyles buniifolius (Hook. ex Arn.) R.M. King \& H. Rob. & $\mathrm{x}$ & $\mathrm{x}$ & - & - & nativa & subarbusto & $\mathrm{x}$ \\
\hline Achyrocline alata (Kunth) DC. & - & - & - & $\mathrm{x}$ & nativa & herbáceo & $\mathrm{x}$ \\
\hline Ambrosia elatior L. & - & $\mathrm{x}$ & - & - & nativa & herbáceo & $\mathrm{x}$ \\
\hline Aspilia montevidensis (Spreng.) Kuntze & $\mathrm{x}$ & $\mathrm{x}$ & - & - & nativa & herbáceo & \\
\hline Baccharis coridifolia DC. & & $\mathrm{x}$ & - & - & nativa & subarbusto & $\mathrm{x}$ \\
\hline B. crispa Spreng & $\mathrm{x}$ & $\mathrm{x}$ & $\mathrm{x}$ & $\mathrm{x}$ & nativa & subarbusto & $\mathrm{x}$ \\
\hline B. dracunculifolia DC. & - & - & - & $\mathrm{x}$ & nativa & arbusto & $\mathrm{x}$ \\
\hline B. junciformis DC. & - & - & - & $\mathrm{x}$ & nativa & subarbusto & $\mathrm{x}$ \\
\hline Chaptalia mandonii Sch.Bip. ex Burkart & $\mathrm{x}$ & $\mathrm{x}$ & - & - & nativa & herbáceo & $\mathrm{x}$ \\
\hline C. runcinata Kunth & - & - & - & $\mathrm{x}$ & nativa & herbáceo & $\mathrm{x}$ \\
\hline Chrysolaena flexuosa (Sims) H. Rob. & - & $\mathrm{x}$ & - & - & nativa & subarbusto & $\mathrm{x}$ \\
\hline Conyza bonariensis (L.) Cronquist & $\mathrm{x}$ & $\mathrm{x}$ & - & $\mathrm{x}$ & nativa & herbáceo & $\mathrm{x}$ \\
\hline C. floribunda Kunth & - & $\mathrm{x}$ & - & - & nativa & herbáceo & $\mathrm{x}$ \\
\hline C. primulifolia (Lam.) Cuatrec. \& Lourteig & $\mathrm{x}$ & - & - & - & nativa & herbáceo & $\mathrm{x}$ \\
\hline Criscia stricta Spreng. & $\mathrm{x}$ & - & - & - & nativa & herbáceo & - \\
\hline Elephantopus mollis Kunth & - & $\mathrm{x}$ & - & - & nativa & herbáceo & - \\
\hline Erechtites hieraciifolius (L.) Raf. ex DC. & - & $\mathrm{x}$ & - & - & nativa & herbáceo & - \\
\hline Facelis retusa (Lam.) Sch. Bip. & $\mathrm{x}$ & $\mathrm{x}$ & - & - & nativa & herbáceo & $\mathrm{x}$ \\
\hline Gamochaeta americana (Mill.) Wedd. & $\mathrm{x}$ & - & - & - & nativa & herbáceo & $\mathrm{x}$ \\
\hline G. coarctata (Willd.) Kerguélen & - & $\mathrm{x}$ & $\mathrm{x}$ & $\mathrm{x}$ & nativa & herbáceo & $\mathrm{x}$ \\
\hline G. falcata (Lam.) Cabrera & $\mathrm{x}$ & - & - & - & nativa & herbáceo & $\mathrm{x}$ \\
\hline G. purpurea (L.) Cabrera & - & - & $\mathrm{x}$ & - & nativa & herbáceo & $\mathrm{x}$ \\
\hline Hypochaeris albiflora (Kuntze) Azevêdo-Gonç. \& Matzenb. & - & $\mathrm{x}$ & - & - & nativa & herbáceo & $\mathrm{x}$ \\
\hline
\end{tabular}


Quadro 1. Continuação

\begin{tabular}{|c|c|c|c|c|c|c|c|}
\hline Famílias/Espécies & CRv & $\mathrm{CSv}$ & CUv & $\mathrm{CBv}$ & Origem & Hábito & LQ \\
\hline H. chillensis (Kunth) Britton & - & $\mathrm{x}$ & - & - & nativa & herbáceo & $\mathrm{x}$ \\
\hline H. glabra L. & - & $\mathrm{x}$ & - & - & nativa & herbáceo & $\mathrm{x}$ \\
\hline H. lutea (Vell.) Britton & - & - & - & $\mathrm{x}$ & nativa & herbáceo & $x$ \\
\hline H. neopinnatifida Azevêdo-Gonç. \& Matzenb. & $\mathrm{x}$ & $\mathrm{x}$ & - & - & nativa & herbáceo & $\mathrm{x}$ \\
\hline Lessingianthus macrocephalus (Less.) H. Rob. & $\mathrm{x}$ & - & - & - & nativa & herbáceo & $\mathrm{x}$ \\
\hline L. sellowii (Less.) H. Rob. & - & $\mathrm{x}$ & - & - & nativa & herbáceo & $\mathrm{x}$ \\
\hline Lucilia nitens Less. & $\mathrm{x}$ & - & - & - & nativa & herbáceo & $x$ \\
\hline Orthopappus angustifolius (Sw.) Gleason & - & $\mathrm{x}$ & - & - & nativa & herbáceo & $\mathrm{x}$ \\
\hline Picrosia longifolia D. Don & - & - & - & $x$ & nativa & herbáceo & - \\
\hline \multicolumn{8}{|l|}{ Asteraceae } \\
\hline Porophyllum linifolium (L.) DC. & $\mathrm{x}$ & - & - & - & nativa & herbáceo & - \\
\hline Pterocaulon cf. balansae Chodat & - & - & - & $\mathrm{x}$ & nativa & subarbusto & $\mathrm{x}$ \\
\hline Pterocaulon sp. 1 & - & $\mathrm{x}$ & - & - & nativa & subarbusto & $\mathrm{x}$ \\
\hline Pterocaulon sp. 2 & - & - & - & $x$ & nativa & subarbusto & $\mathrm{x}$ \\
\hline Senecio brasiliensis (Spreng.) Less. & $\mathrm{x}$ & $x$ & $\mathrm{x}$ & $\mathrm{x}$ & nativa & herbáceo & $\mathrm{x}$ \\
\hline S. heterotrichius DC. & - & - & $\mathrm{x}$ & $\mathrm{x}$ & nativa & subarbusto & $\mathrm{x}$ \\
\hline S. icoglossus DC. & - & - & - & $\mathrm{x}$ & nativa & herbáceo & $\mathrm{x}$ \\
\hline S. leptolobus DC. & $x$ & - & - & - & nativa & subarbusto & $\mathrm{x}$ \\
\hline S. selloi (Spreng.) DC. & - & $\mathrm{x}$ & - & - & nativa & herbáceo & $\mathrm{x}$ \\
\hline Solidago chilensis Meyen & - & $x$ & - & - & nativa & herbáceo & - \\
\hline Soliva sessilis Ruiz et Pavón & $\mathrm{x}$ & $\mathrm{x}$ & $\mathrm{x}$ & - & nativa & herbáceo & $\mathrm{x}$ \\
\hline Sommerfeltia spinulosa Less. & $\mathrm{x}$ & - & - & - & nativa & subarbusto & $\mathrm{x}$ \\
\hline Stenachaenium campestre Baker & $\mathrm{x}$ & - & - & - & nativa & herbáceo & $\mathrm{x}$ \\
\hline S. megapotamicum Baker & - & $\mathrm{x}$ & - & - & nativa & herbáceo & - \\
\hline Symphyotrichum squamatum (Spreng.) G.L. Nesom & - & - & $\mathrm{x}$ & - & nativa & herbáceo & - \\
\hline Vernonanthura nudiflora (Less.) H. Rob. & $\mathrm{x}$ & $\mathrm{x}$ & - & - & nativa & subarbusto & $\mathrm{x}$ \\
\hline \multicolumn{8}{|l|}{ Begoniaceae } \\
\hline Begonia cucullata Will. & - & - & $\mathrm{x}$ & - & nativa & herbáceo & - \\
\hline \multicolumn{8}{|l|}{ Cactaceae } \\
\hline Frailea buenekeri subsp. densispina Hofacker \& Herm & $\mathrm{x}$ & - & - & - & nativa & herbáceo & - \\
\hline Gymnocalycium denudatum (Link \& Otto) Pfeiff. ex Mittler & $\mathrm{x}$ & - & - & - & nativa & herbáceo & - \\
\hline Parodia erinacea (Haw.) N. P. Taylor & $\mathrm{x}$ & - & - & - & nativa & herbáceo & $\mathrm{x}$ \\
\hline P. mammulosa (Lem.) N. P. Taylor & $\mathrm{x}$ & - & - & - & nativa & herbáceo & - \\
\hline P. ottonis (Lehmann) N. P. Taylor & $\mathrm{x}$ & - & - & - & nativa & herbáceo & - \\
\hline \multicolumn{8}{|l|}{ Campanulaceae } \\
\hline Lobelia hederacea Cham. & - & - & $\mathrm{x}$ & $\mathrm{x}$ & nativa & herbáceo & $\mathrm{x}$ \\
\hline Triodanis perfoliata subsp. biflora (Ruiz \& Pav.) Lammers & - & $\mathrm{x}$ & - & - & nativa & herbáceo & $\mathrm{x}$ \\
\hline Wahlenbergia linarioides (Lam.) A. DC. & $\mathrm{x}$ & $\mathrm{x}$ & - & - & nativa & herbáceo & $\mathrm{x}$ \\
\hline \multicolumn{8}{|l|}{ Caryophyllaceae } \\
\hline Cerastium commersonianum Séringe DC. & - & - & - & $\mathrm{x}$ & naturalizada & herbáceo & $\mathrm{x}$ \\
\hline C. glomeratum Thuill. & $\mathrm{x}$ & $\mathrm{x}$ & - & - & naturalizada & herbáceo & $\mathrm{x}$ \\
\hline C. rivulare Cambess. & - & - & - & $\mathrm{x}$ & naturalizada & herbáceo & $\mathrm{x}$ \\
\hline Cerastium sp. & - & - & $\mathrm{x}$ & - & naturalizada & herbáceo & $\mathrm{x}$ \\
\hline Paronychia brasiliana DC. & $\mathrm{x}$ & - & - & - & nativa & herbáceo & $\mathrm{x}$ \\
\hline Polycarpon tetraphyllum (L.) L. & $\mathrm{x}$ & - & - & - & naturalizada & herbáceo & $\mathrm{x}$ \\
\hline \multicolumn{8}{|l|}{ Cistaceae } \\
\hline Helianthemum brasiliense (Lam.) Pers. & $\mathrm{x}$ & - & - & - & nativa & herbáceo & $\mathrm{x}$ \\
\hline Commelinaceae & & & & & & & \\
\hline
\end{tabular}


Quadro 1. Continuação

\begin{tabular}{|c|c|c|c|c|c|c|c|}
\hline Famílias/Espécies & $\mathrm{CRv}$ & $\mathrm{CSv}$ & CUv & $\mathrm{CBv}$ & Origem & Hábito & LQ \\
\hline Commelina platyphylla Klotzsch & - & $\mathrm{x}$ & - & - & nativa & herbáceo & $\mathrm{x}$ \\
\hline \multicolumn{8}{|l|}{ Convolvulaceae } \\
\hline Dichondra macrocalyx Meisn. & - & $\mathrm{x}$ & - & - & nativa & herbáceo & $\mathrm{x}$ \\
\hline D. sericea $\mathrm{Sw}$. & $\mathrm{x}$ & - & - & - & nativa & herbáceo & $\mathrm{x}$ \\
\hline Evolvulus sericeus $\mathrm{Sw}$. & $\mathrm{x}$ & $\mathrm{x}$ & $\mathrm{x}$ & - & nativa & herbáceo & $\mathrm{x}$ \\
\hline \multicolumn{8}{|l|}{ Crassulaceae } \\
\hline Crassula longipes (Rose) M. Bywater \& Wickens & - & - & - & $\mathrm{x}$ & nativa & herbáceo & $\mathrm{x}$ \\
\hline \multicolumn{8}{|l|}{ Cyperaceae } \\
\hline Abildgaardia ovata (Burm. f.) Kral & - & $\mathrm{x}$ & - & - & nativa & herbáceo & $\mathrm{x}$ \\
\hline Bulbostylis capillaris fo. stenantha Kük. ex Barros & $\mathrm{x}$ & $\mathrm{x}$ & - & - & nativa & herbáceo & $\mathrm{x}$ \\
\hline B. juncoides (Vahl) Kük. ex Osten & $\mathrm{x}$ & $\mathrm{x}$ & - & - & nativa & herbáceo & $\mathrm{x}$ \\
\hline Carex bonariensis Desf. ex Poir. & - & - & $\mathrm{x}$ & - & nativa & herbáceo & $\mathrm{x}$ \\
\hline C. longii Mackenz. & - & - & - & $\mathrm{x}$ & nativa & herbáceo & $\mathrm{x}$ \\
\hline C. phalaroides Kunth & - & $\mathrm{x}$ & - & - & nativa & herbáceo & $\mathrm{x}$ \\
\hline C. sororia Kunth & - & $\mathrm{x}$ & - & - & nativa & herbáceo & $\mathrm{x}$ \\
\hline Cyperus aggregatus (Willd.) Endl. & $\mathrm{x}$ & - & - & - & nativa & herbáceo & $\mathrm{x}$ \\
\hline C. reflexus Vahl & - & $\mathrm{x}$ & - & - & nativa & herbáceo & $\mathrm{x}$ \\
\hline C. rigens J. Presl \& C. Presl & - & - & $\mathrm{x}$ & - & nativa & herbáceo & $\mathrm{x}$ \\
\hline Eleocharis bonariensis Nees & - & - & $\mathrm{x}$ & $\mathrm{x}$ & nativa & herbáceo & $\mathrm{x}$ \\
\hline E. sellowiana Kunth & - & - & $\mathrm{x}$ & $\mathrm{x}$ & nativa & herbáceo & $\mathrm{x}$ \\
\hline \multicolumn{8}{|l|}{ Cyperaceae } \\
\hline E. viridans Kük. ex Osten & - & - & $\mathrm{x}$ & $\mathrm{x}$ & nativa & herbáceo & $\mathrm{x}$ \\
\hline Kyllinga brevifolia Rottb. & - & - & $\mathrm{x}$ & - & nativa & herbáceo & $\mathrm{x}$ \\
\hline K. odorata Vahl & - & $\mathrm{x}$ & $\mathrm{x}$ & - & nativa & herbáceo & $\mathrm{x}$ \\
\hline Pycreus megapotamicus (Kunth) Nees & - & - & - & $\mathrm{x}$ & nativa & herbáceo & $\mathrm{x}$ \\
\hline P. polystachyos (Rottb.) P. Beauv. & - & - & $\mathrm{x}$ & $\mathrm{x}$ & nativa & herbáceo & - \\
\hline Rhynchospora brownii Roem. \& Schult. & - & - & $\mathrm{x}$ & - & nativa & herbáceo & - \\
\hline R. conferta (Nees) Boeckeler & - & - & - & $\mathrm{x}$ & nativa & herbáceo & $\mathrm{x}$ \\
\hline R. scutellata Griseb. & - & - & - & $\mathrm{x}$ & nativa & herbáceo & $\mathrm{x}$ \\
\hline R. marisculus Lindl. \& Nees & - & - & - & $\mathrm{x}$ & nativa & herbáceo & $\mathrm{x}$ \\
\hline R. megapotamica (Spreng.) H. Pfeiff. & - & - & $\mathrm{x}$ & - & nativa & herbáceo & - \\
\hline R. tenuis Link & - & - & $\mathrm{x}$ & - & nativa & herbáceo & - \\
\hline \multicolumn{8}{|l|}{ Euphorbiaceae } \\
\hline Acalypha communis Müll. Arg. & $\mathrm{x}$ & - & - & - & nativa & subarbusto & - \\
\hline Croton sp. & $\mathrm{x}$ & - & - & - & nativa & subarbusto & $\mathrm{x}$ \\
\hline Ditaxis acaulis Herter ex Arechav. & $\mathrm{x}$ & - & - & - & nativa & herbáceo & $\mathrm{x}$ \\
\hline Euphorbia selloi (Klotzsch \& Garcke) Boiss & $\mathrm{x}$ & - & - & - & nativa & herbáceo & $\mathrm{x}$ \\
\hline \multicolumn{8}{|l|}{ Fabaceae } \\
\hline Desmodium incanum DC. & $\mathrm{x}$ & $\mathrm{x}$ & - & - & nativa & subarbusto & $\mathrm{x}$ \\
\hline Galactia marginalis Benth. ex Benth. \& Hook. f. & $\mathrm{x}$ & $\mathrm{x}$ & - & - & nativa & subarbusto & $\mathrm{x}$ \\
\hline Lathyrus subulatus Lam. & - & $\mathrm{x}$ & - & - & nativa & herbáceo & - \\
\hline Lupinus linearis Desr. & $\mathrm{x}$ & - & - & - & nativa & herbáceo & - \\
\hline Mimosa flagellaris Benth. & - & $\mathrm{x}$ & - & - & nativa & subarbusto & $\mathrm{x}$ \\
\hline Pomaria pilosa (Vogel) B.B.Simpson \& G.P.Lewis & $\mathrm{x}$ & - & - & - & nativa & subarbusto & $\mathrm{x}$ \\
\hline Rhynchosia diversifolia Micheli & $\mathrm{x}$ & - & - & - & nativa & herbáceo & $\mathrm{x}$ \\
\hline Stylosanthes leiocarpa Vogel & $\mathrm{x}$ & - & - & - & nativa & herbáceo & $\mathrm{x}$ \\
\hline S. montevidensis Vogel & $\mathrm{x}$ & - & - & - & nativa & herbáceo & $\mathrm{x}$ \\
\hline Trifolium polymorphum Poir. & $\mathrm{x}$ & $\mathrm{x}$ & $\mathrm{x}$ & - & nativa & herbáceo & $\mathrm{x}$ \\
\hline
\end{tabular}


Quadro 1. Continuação

\begin{tabular}{|c|c|c|c|c|c|c|c|}
\hline Famílias/Espécies & CRv & $\mathrm{CSv}$ & CUv & $\mathrm{CBv}$ & Origem & Hábito & LQ \\
\hline Zornia sp. & $\mathrm{x}$ & - & - & - & nativa & herbáceo & $\mathrm{x}$ \\
\hline \multicolumn{8}{|l|}{ Gentianaceae } \\
\hline Zygostigma australe (Cham. \& Schltdl.) Griseb. & $\mathrm{x}$ & - & - & - & nativa & herbáceo & $\mathrm{x}$ \\
\hline \multicolumn{8}{|l|}{ Hypoxidaceae } \\
\hline Hypoxis decumbens $\mathrm{L}$. & - & $\mathrm{x}$ & $\mathrm{x}$ & $\mathrm{x}$ & nativa & herbáceo & $\mathrm{x}$ \\
\hline \multicolumn{8}{|l|}{ Iridaceae } \\
\hline Cypella pusilla Benth. \& Hook.f. & $\mathrm{x}$ & - & - & - & nativa & herbáceo & - \\
\hline Herbertia lahue (Molina) Goldblatt & $\mathrm{x}$ & $\mathrm{x}$ & - & - & nativa & herbáceo & $\mathrm{x}$ \\
\hline Kelissa brasiliensis (Baker) Ravenna & $\mathrm{x}$ & $\mathrm{x}$ & - & - & nativa & herbáceo & $\mathrm{x}$ \\
\hline Sisyrinchium megapotamicum Malme & - & $\mathrm{x}$ & - & - & nativa & herbáceo & $\mathrm{x}$ \\
\hline S. micranthum Cav. & $\mathrm{x}$ & $\mathrm{x}$ & $\mathrm{x}$ & - & nativa & herbáceo & $\mathrm{x}$ \\
\hline S. platense I. M. Johnst. & - & - & - & $\mathrm{x}$ & nativa & herbáceo & $\mathrm{x}$ \\
\hline \multicolumn{8}{|l|}{ Juncaceae } \\
\hline Juncus capillaceus Lam. & - & $\mathrm{x}$ & $\mathrm{x}$ & - & nativa & herbáceo & $\mathrm{x}$ \\
\hline J. microcephalus Kunth & - & - & $\mathrm{x}$ & $\mathrm{x}$ & nativa & herbáceo & $\mathrm{x}$ \\
\hline J. tenuis Willd. & - & - & $\mathrm{x}$ & - & nativa & herbáceo & $\mathrm{x}$ \\
\hline \multicolumn{8}{|l|}{ Lamiaceae } \\
\hline Hyptis mutabilis Briq. & $x$ & $\mathrm{x}$ & - & - & nativa & subarbusto & $\mathrm{x}$ \\
\hline Salvia procurrens Benth. & - & $\mathrm{x}$ & - & - & nativa & herbáceo & - \\
\hline Scutellaria racemosa Pers. & - & - & $\mathrm{x}$ & $x$ & nativa & herbáceo & $\mathrm{x}$ \\
\hline \multicolumn{8}{|l|}{ Lentibulariaceae } \\
\hline Utricularia tricolor A. St.-Hil. & - & - & - & $\mathrm{x}$ & nativa & herbáceo & - \\
\hline \multicolumn{8}{|l|}{ Linaceae } \\
\hline $\begin{array}{l}\text { Cliococca selaginoides (Lam.) C. M. Rogers \& Mild } \\
\text { Loganiaceae }\end{array}$ & \multicolumn{6}{|c|}{ Loganiaceae } & $\mathrm{x}$ \\
\hline Spigelia stenophylla Progel & $\mathrm{x}$ & - & - & - & nativa & herbáceo & - \\
\hline \multicolumn{8}{|l|}{ Lythraceae } \\
\hline Cuphea glutinosa Cham. \& Schltdl. & $\mathrm{x}$ & $\mathrm{x}$ & $\mathrm{x}$ & - & nativa & herbáceo & $\mathrm{x}$ \\
\hline C. racemosa (L.f.) Spreng. & - & - & $\mathrm{x}$ & - & nativa & herbáceo & - \\
\hline \multicolumn{8}{|l|}{ Malvaceae } \\
\hline $\begin{array}{l}\text { Ayenia mansfeldiana (Herter) Herter ex Cristóbal } \\
\text { Malvaceae }\end{array}$ & $\mathrm{x}$ & - & - & - & nativa & herbáceo & $\mathrm{x}$ \\
\hline Krapovickasia flavescens (Cav.) Fryxell & $\mathrm{x}$ & - & - & - & nativa & herbáceo & $\mathrm{x}$ \\
\hline Sida rhombifolia L. & - & $x$ & - & - & nativa & subarbusto & $\mathrm{x}$ \\
\hline \multicolumn{8}{|l|}{ Melastomataceae } \\
\hline $\begin{array}{l}\text { Tibouchina gracilis (Bonpl.) Cogn. } \\
\text { Moraceae }\end{array}$ & - & - & - & $\mathrm{x}$ & nativa & herbáceo & $\mathrm{x}$ \\
\hline $\begin{array}{l}\text { Dorstenia brasiliensis Lam. } \\
\text { Onagraceae }\end{array}$ & $\mathrm{x}$ & - & - & - & nativa & herbáceo & $\mathrm{x}$ \\
\hline Ludwigia hookeri (Micheli) H. Hara & - & - & $\mathrm{x}$ & $\mathrm{x}$ & nativa & herbáceo & - \\
\hline Oenothera sp. & $\mathrm{x}$ & $\mathrm{x}$ & - & - & nativa & herbáceo & $\mathrm{x}$ \\
\hline \multicolumn{8}{|l|}{ Orchidaceae } \\
\hline Brachystele camporum (Lindl.) Schltr. & $\mathrm{x}$ & - & - & - & nativa & herbáceo & $\mathrm{x}$ \\
\hline Habenaria parviflora Lindl. & - & - & $\mathrm{x}$ & $\mathrm{x}$ & nativa & herbáceo & - \\
\hline Habenaria sp. & - & - & - & $\mathrm{x}$ & nativa & herbáceo & - \\
\hline \multicolumn{8}{|l|}{ Oxalidaceae } \\
\hline Oxalis articulata Savigny & - & $\mathrm{x}$ & - & - & nativa & herbáceo & $\mathrm{x}$ \\
\hline O. brasiliensis Lodd. & - & $\mathrm{x}$ & $\mathrm{x}$ & - & nativa & herbáceo & $\mathrm{x}$ \\
\hline
\end{tabular}


Quadro 1. Continuação

\begin{tabular}{|c|c|c|c|c|c|c|c|}
\hline Famílias/Espécies & CRv & $\mathrm{CSv}$ & CUv & $\mathrm{CBv}$ & Origem & Hábito & LQ \\
\hline O. conorrhiza Jacq. & $\mathrm{x}$ & $\mathrm{x}$ & - & - & nativa & herbáceo & $\mathrm{x}$ \\
\hline O. eriocarpa DC. & $\mathrm{x}$ & $\mathrm{x}$ & - & - & nativa & herbáceo & $\mathrm{x}$ \\
\hline O. lasiopetala Zucc. & - & $\mathrm{x}$ & - & - & nativa & herbáceo & $\mathrm{x}$ \\
\hline O. sellowiana Zucc. & $\mathrm{x}$ & $x$ & - & - & nativa & herbáceo & - \\
\hline \multicolumn{8}{|l|}{ Passifloraceae } \\
\hline Piriqueta taubatensis (Urb.) Arbo & - & $\mathrm{x}$ & - & - & nativa & herbáceo & - \\
\hline Turnera sidoides L. & $\mathrm{x}$ & - & - & - & nativa & herbáceo & $\mathrm{x}$ \\
\hline \multicolumn{8}{|l|}{ Plantaginaceae } \\
\hline Bacopa sp. & - & $\mathrm{x}$ & $\mathrm{x}$ & - & nativa & herbáceo & $\mathrm{x}$ \\
\hline Plantago tomentosa Lam. & $\mathrm{x}$ & $\mathrm{x}$ & - & - & nativa & herbáceo & $\mathrm{x}$ \\
\hline Scoparia montevidensis (Spreng.) R. E. Fr. & $\mathrm{x}$ & $\mathrm{x}$ & - & - & nativa & herbáceo & - \\
\hline \multicolumn{8}{|l|}{ Poaceae } \\
\hline Andropogon lateralis Nees & $x$ & $\mathrm{x}$ & $x$ & $x$ & nativa & herbáceo & $\mathrm{x}$ \\
\hline A. ternatus (Spreng.) Nees & $\mathrm{x}$ & $\mathrm{x}$ & - & $\mathrm{x}$ & nativa & herbáceo & $\mathrm{x}$ \\
\hline A. virgatus Desv. ex Ham. & - & - & - & $x$ & nativa & herbáceo & $\mathrm{x}$ \\
\hline Aristida jubata (Arechav.) Herter & $\mathrm{x}$ & $\mathrm{x}$ & - & - & nativa & herbáceo & $\mathrm{x}$ \\
\hline A. laevis (Nees) Kunth & $\mathrm{x}$ & - & - & - & nativa & herbáceo & $x$ \\
\hline A. murina Cav. & $\mathrm{x}$ & $\mathrm{x}$ & - & - & nativa & herbáceo & $\mathrm{x}$ \\
\hline A. venustula Arechav. & $x$ & $\mathrm{x}$ & $x$ & - & nativa & herbáceo & $\mathrm{x}$ \\
\hline Axonopus affinis Chase & - & $\mathrm{x}$ & $\mathrm{x}$ & - & nativa & herbáceo & $\mathrm{x}$ \\
\hline A. suffultus (Mikan ex Trin.) Parodi & $x$ & $x$ & $x$ & - & nativa & herbáceo & $\mathrm{x}$ \\
\hline Bothriochloa laguroides (DC.) Herter & $\mathrm{x}$ & $\mathrm{x}$ & $\mathrm{x}$ & - & nativa & herbáceo & $\mathrm{x}$ \\
\hline Briza minor L. & - & $\mathrm{x}$ & $\mathrm{x}$ & $\mathrm{x}$ & naturalizada & herbáceo & $\mathrm{x}$ \\
\hline $\begin{array}{l}\text { Chascolytrum poomorphum (J. Presl) Essi, Longhi-Wagner } \\
\text { \& Souza-Chies }\end{array}$ & - & - & $\mathrm{x}$ & $\mathrm{x}$ & nativa & herbáceo & $\mathrm{x}$ \\
\hline C. rufum J. Presl & - & - & $\mathrm{x}$ & - & nativa & herbáceo & $\mathrm{x}$ \\
\hline C. subaristatum (Lam.) Desv. & $x$ & $\mathrm{x}$ & $\mathrm{x}$ & - & nativa & herbáceo & $\mathrm{x}$ \\
\hline C. uniolae (Nees) Essi, Longhi-Wagner \& Souza-Chies & - & - & - & $x$ & nativa & herbáceo & $\mathrm{x}$ \\
\hline Danthonia montevidensis Hack. \& Arechav. & $\mathrm{x}$ & $\mathrm{x}$ & - & - & nativa & herbáceo & $\mathrm{x}$ \\
\hline Dichanthelium sabulorum (Lam.) Gould \& C. A. Clark & - & $\mathrm{x}$ & - & - & nativa & herbáceo & $\mathrm{x}$ \\
\hline Eleusine tristachya (Lam.) Lam. & $\mathrm{x}$ & $\mathrm{x}$ & - & - & nativa & herbáceo & $\mathrm{x}$ \\
\hline Elionurus sp. & $\mathrm{x}$ & - & - & - & nativa & herbáceo & $\mathrm{x}$ \\
\hline Eragrostis bahiensis Schrad. & - & $\mathrm{x}$ & - & - & nativa & herbáceo & - \\
\hline E. lugens Nees & $\mathrm{x}$ & $\mathrm{x}$ & - & - & nativa & herbáceo & $\mathrm{x}$ \\
\hline E. neesii Trin. & $\mathrm{x}$ & $\mathrm{x}$ & - & - & nativa & herbáceo & $\mathrm{x}$ \\
\hline Leersia hexandra $\mathrm{Sw}$. & - & - & $\mathrm{x}$ & $x$ & nativa & herbáceo & $\mathrm{x}$ \\
\hline Lolium multiflorum Lam. & - & $\mathrm{x}$ & $\mathrm{x}$ & - & exótica & herbáceo & $\mathrm{x}$ \\
\hline Luziola peruviana Juss. ex J. F. Gmel. & - & - & $\mathrm{x}$ & $\mathrm{x}$ & nativa & herbáceo & $\mathrm{x}$ \\
\hline Melica rigida Cav. & - & $\mathrm{x}$ & - & - & nativa & herbáceo & $\mathrm{x}$ \\
\hline Microchloa indica (L. f.) P. Beauv. & $\mathrm{x}$ & - & - & - & nativa & herbáceo & $\mathrm{x}$ \\
\hline Mnesithea selloana (Hack.) de Koning \& Sosef & $\mathrm{x}$ & $x$ & - & - & nativa & herbáceo & $\mathrm{x}$ \\
\hline Paspalum dilatatum Poir. & - & - & $\mathrm{x}$ & $\mathrm{x}$ & nativa & herbáceo & $\mathrm{x}$ \\
\hline \multicolumn{8}{|l|}{ Poaceae } \\
\hline P. exaltatum J. Presl & - & - & - & $\mathrm{x}$ & nativa & herbáceo & $x$ \\
\hline P. lepton Schult. & - & $\mathrm{x}$ & $x$ & - & nativa & herbáceo & $x$ \\
\hline P. maculosum Trin. & - & $\mathrm{x}$ & - & - & nativa & herbáceo & $\mathrm{x}$ \\
\hline P. notatum Fluegge & $\mathrm{x}$ & $\mathrm{x}$ & $\mathrm{x}$ & - & nativa & herbáceo & $\mathrm{x}$ \\
\hline P. plicatulum Michx. & $\mathrm{x}$ & $\mathrm{x}$ & - & - & nativa & herbáceo & $\mathrm{x}$ \\
\hline
\end{tabular}


Quadro 1. Continuação

\begin{tabular}{|c|c|c|c|c|c|c|c|}
\hline Famílias/Espécies & $\mathrm{CRv}$ & $\mathrm{CSv}$ & $\mathrm{CUv}$ & $\mathrm{CBv}$ & Origem & Hábito & LQ \\
\hline P. pumilum Nees & - & - & $\mathrm{x}$ & - & nativa & herbáceo & $\mathrm{x}$ \\
\hline P. vaginatum $\mathrm{Sw}$. & - & - & $\mathrm{x}$ & - & nativa & herbáceo & $\mathrm{x}$ \\
\hline Phalaris angusta Nees ex Trin. & - & - & $\mathrm{x}$ & - & nativa & herbáceo & $\mathrm{x}$ \\
\hline Piptochaetium lasianthum Griseb. & $\mathrm{x}$ & - & - & - & nativa & herbáceo & $\mathrm{x}$ \\
\hline P. montevidense (Spreng.) Parodi & $\mathrm{x}$ & $\mathrm{x}$ & - & - & nativa & herbáceo & $\mathrm{x}$ \\
\hline P. stipoides (Trin. \& Rupr.) Hack. & $\mathrm{x}$ & $\mathrm{x}$ & - & - & nativa & herbáceo & $\mathrm{x}$ \\
\hline Poa annua $\mathrm{L}$. & - & - & $\mathrm{x}$ & - & naturalizada & herbáceo & $\mathrm{x}$ \\
\hline Saccharum angustifolium Trin & - & $\mathrm{x}$ & - & - & nativa & herbáceo & - \\
\hline S. villosum Steud. & - & - & - & $\mathrm{x}$ & nativa & herbáceo & $\mathrm{x}$ \\
\hline Schizachyrium microstachyum (Desv. ex Ham.) Roseng. & - & $\mathrm{x}$ & - & - & nativa & herbáceo & $\mathrm{x}$ \\
\hline S. spicatum (Spreng.) Herter & $\mathrm{x}$ & - & - & - & nativa & herbáceo & $\mathrm{x}$ \\
\hline Setaria parviflora (Poir.) Kerguélen & $\mathrm{x}$ & $\mathrm{x}$ & - & - & nativa & herbáceo & $\mathrm{x}$ \\
\hline Sporobolus indicus (L.) R. Br. & $\mathrm{x}$ & $\mathrm{x}$ & $\mathrm{x}$ & - & nativa & herbáceo & $\mathrm{x}$ \\
\hline Steinchisma decipiens (Nees ex Trin.) W. V. Br. & - & - & $\mathrm{x}$ & - & nativa & herbáceo & $\mathrm{x}$ \\
\hline S. hians (Elliott) Nash & - & $\mathrm{x}$ & - & - & nativa & herbáceo & $\mathrm{x}$ \\
\hline Stipa setigera J. Presl & $\mathrm{x}$ & $\mathrm{x}$ & - & - & nativa & herbáceo & $\mathrm{x}$ \\
\hline Trachypogon montufari Ness & - & $\mathrm{x}$ & - & - & nativa & herbáceo & - \\
\hline T. montufari Ness var. mollis (Ness) Burkart & $\mathrm{x}$ & - & - & - & nativa & herbáceo & $\mathrm{x}$ \\
\hline Trichanthecium schwackeanum (Mez) Zuloaga \& Morrone & - & - & $\mathrm{x}$ & - & nativa & herbáceo & $\mathrm{x}$ \\
\hline Vulpia bromoides (L.) Gray & $\mathrm{x}$ & $\mathrm{x}$ & - & - & naturalizada & herbáceo & $\mathrm{x}$ \\
\hline \multicolumn{8}{|l|}{ Polygalaceae } \\
\hline Polygala australis A. W. Benn. & $\mathrm{x}$ & - & - & - & nativa & herbáceo & $\mathrm{x}$ \\
\hline P. linoides Poir. & $\mathrm{x}$ & - & - & - & nativa & herbáceo & $\mathrm{x}$ \\
\hline \multicolumn{8}{|l|}{ Polygonaceae } \\
\hline Polygonum punctatum Elliott & - & - & $\mathrm{x}$ & $\mathrm{x}$ & nativa & herbáceo & $\mathrm{x}$ \\
\hline \multicolumn{8}{|l|}{ Pontederiaceae } \\
\hline \multicolumn{7}{|l|}{ Primulaceae } & - \\
\hline Anagallis arvensis L. & - & $\mathrm{x}$ & - & - & naturalizada & herbáceo & - \\
\hline \multicolumn{8}{|l|}{ Ranunculaceae } \\
\hline Ranunculus bonariensis Poir. & - & - & $\mathrm{x}$ & - & nativa & herbáceo & $\mathrm{x}$ \\
\hline \multicolumn{8}{|l|}{ Rubiaceae } \\
\hline $\begin{array}{l}\text { Borreria dasycephala (Cham. \& Schltdl.) Bacigalupo \& } \\
\text { E.L.Cabral }\end{array}$ & - & $\mathrm{x}$ & - & - & nativa & herbáceo & $\mathrm{x}$ \\
\hline B. verticillata (L.) G. Mey. & - & $\mathrm{x}$ & - & - & nativa & herbáceo & $x$ \\
\hline Galianthe fastigiata Griseb. & $\mathrm{x}$ & $\mathrm{x}$ & - & - & nativa & herbáceo & $\mathrm{x}$ \\
\hline Galium hirtum Lam. & $\mathrm{x}$ & - & $\mathrm{x}$ & $\mathrm{x}$ & nativa & herbáceo & $\mathrm{x}$ \\
\hline G. noxium (A. St. - Hil.) Dempster & - & - & - & $\mathrm{x}$ & nativa & herbáceo & - \\
\hline G. richardianum (Gillies ex Hook. \& Arn.) Endl. ex Walp. & $\mathrm{x}$ & $\mathrm{x}$ & - & - & nativa & herbáceo & $\mathrm{x}$ \\
\hline Richardia humistrata (Cham. et Schlecht.) Steud. & $\mathrm{x}$ & $\mathrm{x}$ & - & - & nativa & herbáceo & $\mathrm{x}$ \\
\hline R. stellaris (Cham. \& Schltdl.) Steud. & $\mathrm{x}$ & $\mathrm{x}$ & - & - & nativa & herbáceo & $x$ \\
\hline \multicolumn{8}{|l|}{ Schrophulariaceae } \\
\hline Scrophularia peregrina $\mathrm{L}$. & - & - & $\mathrm{x}$ & - & naturalizada & herbáceo & $\mathrm{x}$ \\
\hline \multicolumn{8}{|l|}{ Solanaceae } \\
\hline Nierembergia cf. riograndensis Hunz. \& A.A. Cocucci & - & $\mathrm{x}$ & - & - & nativa & herbáceo & $\mathrm{x}$ \\
\hline Solanum hasslerianum Chodat & $\mathrm{x}$ & - & - & - & nativa & herbáceo & $\mathrm{x}$ \\
\hline \multicolumn{8}{|l|}{ Verbenaceae } \\
\hline Aloysia lycioides Cham. & - & $\mathrm{x}$ & - & - & nativa & subarbusto & $\mathrm{x}$ \\
\hline
\end{tabular}


Quadro 1. Continuação

\begin{tabular}{|c|c|c|c|c|c|c|c|}
\hline Famílias/Espécies & $\mathrm{CRv}$ & $\mathrm{CSv}$ & CUv & $\mathrm{CBv}$ & Origem & Hábito & LQ \\
\hline Glandularia selloi (Spreng.) Tronc. & - & $\mathrm{x}$ & - & - & nativa & herbáceo & - \\
\hline G. thymoides (Cham.) N.O'Leary & $\mathrm{x}$ & - & - & - & nativa & herbáceo & - \\
\hline Verbena rigida Spreng. & - & $\mathrm{x}$ & - & - & nativa & herbáceo & - \\
\hline V. montevidensis Spreng. & - & $\mathrm{x}$ & $\mathrm{x}$ & - & nativa & herbáceo & $\mathrm{x}$ \\
\hline \multicolumn{8}{|l|}{ Violaceae } \\
\hline Hybanthus bicolor (Saint-Hilaire) Baill. & - & $\mathrm{x}$ & - & - & nativa & herbáceo & - \\
\hline \multicolumn{8}{|l|}{ Xyridaceae } \\
\hline Xyris jupicai Rich & - & - & - & $\mathrm{x}$ & nativa & herbáceo & $\mathrm{x}$ \\
\hline Xyris sp. & - & - & - & $\mathrm{x}$ & nativa & herbáceo & $\mathrm{x}$ \\
\hline \multicolumn{8}{|l|}{ Licófita } \\
\hline \multicolumn{8}{|l|}{ Selaginellaceae } \\
\hline Selaginella sp. & - & - & - & $\mathrm{x}$ & nativa & herbáceo & - \\
\hline \multicolumn{8}{|l|}{ Monilófitas } \\
\hline \multicolumn{8}{|l|}{ Pteridaceae } \\
\hline Adiantopsis chlorophylla (Sw.) Fée & - & - & - & $\mathrm{x}$ & nativa & herbáceo & - \\
\hline \multicolumn{8}{|l|}{ Thelypteridaceae } \\
\hline Thelypteris sp. & - & - & - & $\mathrm{x}$ & nativa & herbáceo & $\mathrm{x}$ \\
\hline
\end{tabular}

Paspalum dilatatum e Hydrocotyle ranunculoides (Tab. 2C). No campo brejoso, prevaleceram: Eleocharis viridans, Rhynchospora conferta, $R$. marisculus, acompanhadas de Andropogon lateralis, Hygrophila costata, Chascolytrum uniolae, Rhynchospora scutellata e Baccharis crispa (Tab. 2D). Considerando os valores cumulativos de porcentagem dos IVI de todas as espécies das famílias Poaceae e Cyperaceae, nas fitofisionomias do campo úmido e do campo brejoso, as mesmas contabilizaram 70 e $63 \%$ em cada ambiente, respectivamente (Figs. 4C, D).

Com relação à família Fabaceae, esta família teve maior FR nos campos rupestre e seco, com destaque em ordem decrescente de IVI às espécies Desmodium incanum, Rhynchosia diversifolia e Trifolium polymorphum (Tabs. 2A, B). No campo úmido, Fabaceae foi pouco expressiva, sendo representada unicamente por T. polymorphum e com baixo valor de FR e CR (Tab. 2C). No campo brejoso, tanto no inventário florístico, quanto no levantamento quantitativo não houve registro de leguminosas.

A média de espécies levantadas por UA no campo rupestre foi de 29,1 (desvio padrão $\pm 6,7$ ); no campo seco $31,5( \pm 5,1)$; no campo úmido $16,9( \pm 3,5)$; no campo brejoso $15,5( \pm 3,8)$. Em geral, estes valores foram superiores ao evidenciado por Setubal \& Boldrini (2012), com exceção do campo úmido. Neste sentido, cabe salientar os autores citados registraram no campo rupestre uma média de 23,6 spp. por UA, no campo seco, 22,1 spp. e, no campo brejoso, 7,6 spp.

Pela aplicação do índice de Jaccard (Tab. 3), houve maior similaridade entre o campo rupestre e o campo seco $(0,35)$ e entre campo úmido e campo brejoso $(0,23)$. A maior similaridade entre as áreas com menor saturação hídrica reforça o filtro ambiental atuante nas fitofisionomias de campo rupestre e seco. Áreas em condições mais extremas possuem filtros ambientais que atuam selecionando espécies capazes de colonizar condições adversas (Keddy 1992). Com relação ao índice de Shannon-Wiener, o ambiente com maior diversidade foi o campo seco (4,36 Nats), seguido pelo campo rupestre (4,35 Nats), campo úmido (3,77 Nats) e, por último, o campo brejoso (3,73 Nats). As duas fitofisionomias com maior diversidade e riqueza foram as áreas com menor saturação hídrica, seguindo o padrão encontrado por Setubal \& Boldrini (2012).

A análise de PCoA mostrou um agrupamento das UAs em quatro comunidades campestres (Fig. 5). Os dois primeiros eixos da ordenação contabilizaram 31\% da variância total. Dentre todos os táxons incluídos na matriz de dados, somente 33 apresentaram maior correlação com os eixos 1 e 2 da ordenação. A porção positiva reuniu as UAs de campo seco e rupestre, enquanto a porção negativa agregou as UAs do campo úmido e brejoso. As espécies com os maiores coeficientes de correlação na porção positiva do eixo 1 foram: Paspalum notatum $(0,7)$, Vernonanthura nudiflora (0,69), Eryngium horridum $(0,69)$, Mnesithea selloana (0,69), Vulpia bromoides $(0,65)$, Hypochaeris neopinnatifida (0,63), Desmodium incanum (0,61), Piptochaetium montevidense $(0,60)$, Setaria parviflora $(0,58)$, Galium richardianum $(0,58)$, Stenachaenium campestre (0,53), Andropogon ternatus $(0,53)$, Evolvulus sericeus $(0,53)$, Richardia humistrata $(0,52)$, Wahlenbergia linarioides (0,52), Dichondra sericea (0,51), Stipa setigera $(0,50)$ e Rhynchosia diversifolia $(0,50)$; na porção negativa do Eixo 1, destacaram-se: Bacopa sp. (-0,62), Luziola peruviana (-0,61), Eleocharis bonariensis (-0,61), Paspalum pumilum (-0,61), Paspalum dilatatum (-0,58), Eleocharis viridans $(-0,57)$, Lobelia hederacea $(-0,52)$, 

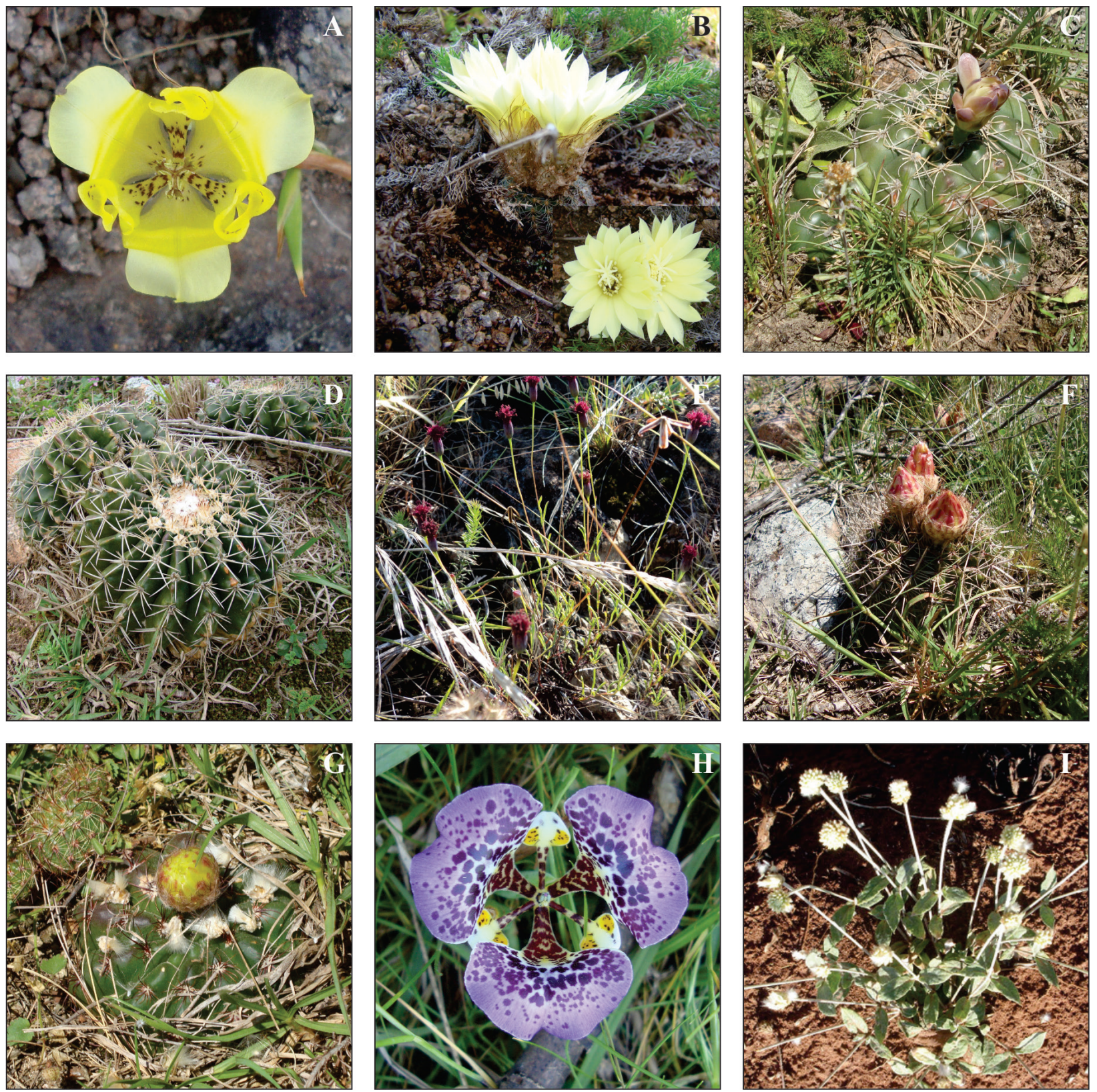

Figs. 3A-I. Espécies registradas na área de estudo citadas como ameaçadas de extinção em diferentes categorias. A. Cypella pusilla, como criticamente em perigo; B. Frailea buenekeri subsp. densispina; C. Gymnocalycium denudatum; D. Parodia erinacea; E. Porophyllum linifolium, em perigo; F. Parodia mammulosa; G. Parodia ottonis; H. Kelissa brasiliensis, como vulnerável; I. Pfaffia gnaphaloides, como quase ameaçada.

Tabela 1. Riqueza específica (spp.) documentada em trabalhos fitossociológicos nos Campos arbustivos do Rio Grande do Sul.

\begin{tabular}{lcc}
\hline Município/Localidade & Referências & Riqueza específica \\
\hline São Gabriel (cabeceiras do Arroio do Salso) & Este trabalho & 199 \\
Lavras do Sul (Mina Volta Grande) & Zocche (2002) & 171 \\
Lavras do Sul (Mina Volta Grande) & Sippel (2003) & 72 \\
Lavras do Sul (Mina Volta Grande) & Frizzo \& Porto (2004) & 106 \\
Porto Alegre (Morro da Polícia) & Boldrini et al. (1998) & 189 \\
Porto Alegre (Morro Santana) & Overbeck et al. (2006) & 221 \\
Porto Alegre (Morro do Osso) & Ferreira et al. (2010) & 161 \\
Porto Alegre (Morro São Pedro) & Setubal \& Boldrini (2012) & 177 \\
Porto Alegre (Jardim Botânico de Porto Alegre) & Dresseno \& Overbeck (2013) & 170 \\
Porto Alegre (Jardim Botânico de Porto Alegre) & Rolim et al. (2014) & 109 \\
Canguçu (Cabanha Sobrado Branco) & Caporal \& Boldrini (2007) & 173 \\
\hline
\end{tabular}


Tabela 2A. Parâmetros quantitativos avaliados no campo rupestre. $\mathrm{FA}=$ frequência absoluta; $\mathrm{FR}=$ frequência relativa; $\mathrm{CA}=$ cobertura absoluta; $\mathrm{CR}=$ cobertura relativa e IVI = índice de valor de importância.

\begin{tabular}{|c|c|c|c|c|c|c|}
\hline Campo rupestre/Táxons & Famílias & FA & FR & $\mathrm{CA}$ & $\mathrm{CR}$ & IVI \\
\hline Aristida jubata & Poaceae & 50 & 1,72 & 115 & 10,28 & 6,00 \\
\hline Aristida venustula & Poaceae & 90 & 3,09 & 66 & 5,90 & 4,50 \\
\hline Baccharis crispa & Asteraceae & 80 & 2,75 & 62 & 5,54 & 4,14 \\
\hline Paspalum notatum & Poaceae & 80 & 2,75 & 50 & 4,47 & 3,61 \\
\hline Eryngium horridum & Apiaceae & 50 & 1,72 & 60 & 5,36 & 3,54 \\
\hline Vernonanthura nudiflora & Asteraceae & 80 & 2,75 & 44 & 3,93 & 3,34 \\
\hline Trachypogon montufari var. mollis & Poaceae & 50 & 1,72 & 45 & 4,02 & 2,87 \\
\hline Stenachaenium campestre & Asteraceae & 60 & 2,06 & 40 & 3,57 & 2,82 \\
\hline Piptochaetium montevidense & Poaceae & 50 & 1,72 & 42 & 3,75 & 2,74 \\
\hline Vulpia bromoides & Poaceae & 70 & 2,41 & 28 & 2,50 & 2,45 \\
\hline Mnesithea selloana & Poaceae & 50 & 1,72 & 35 & 3,13 & 2,42 \\
\hline Andropogon ternatus & Poaceae & 60 & 2,06 & 31 & 2,77 & 2,42 \\
\hline Sommerfeltia spinulosa & Asteraceae & 60 & 2,06 & 27 & 2,41 & 2,24 \\
\hline Cerastium glomeratum & Caryophyllaceae & 70 & 2,41 & 20 & 1,79 & 2,10 \\
\hline Helianthemum brasiliense & Cistaceae & 70 & 2,41 & 19 & 1,70 & 2,05 \\
\hline Evolvulus sericeus & Convolvulaceae & 70 & 2,41 & 16 & 1,43 & 1,92 \\
\hline Hypochaeris neopinnatifida & Asteraceae & 70 & 2,41 & 15 & 1,34 & 1,87 \\
\hline Dichondra sericea & Convolvulaceae & 40 & 1,37 & 26 & 2,32 & 1,85 \\
\hline Ayenia mansfeldiana & Malvaceae & 50 & 1,72 & 22 & 1,97 & 1,84 \\
\hline Rhynchosia diversifolia & Fabaceae & 70 & 2,41 & 11 & 0,98 & 1,69 \\
\hline Paspalum plicatulum & Poaceae & 30 & 1,03 & 26 & 2,32 & 1,68 \\
\hline Sisyrinchium micranthum & Iridaceae & 50 & 1,72 & 18 & 1,61 & 1,66 \\
\hline Chascolytrum subaristatum & Poaceae & 50 & 1,72 & 18 & 1,61 & 1,66 \\
\hline Kelissa brasiliensis & Iridaceae & 50 & 1,72 & 9 & 0,80 & 1,26 \\
\hline Axonopus affinis & Poaceae & 50 & 1,72 & 9 & 0,80 & 1,26 \\
\hline Eragrostis neesii & Poaceae & 40 & 1,37 & 12 & 1,07 & 1,22 \\
\hline Acanthostyles buniifolius & Asteraceae & 30 & 1,03 & 12 & 1,07 & 1,05 \\
\hline Gamochaeta americana & Asteraceae & 40 & 1,37 & 8 & 0,71 & 1,04 \\
\hline Krapovickasia flavescens & Malvaceae & 40 & 1,37 & 8 & 0,71 & 1,04 \\
\hline Danthonia montevidensis & Poaceae & 40 & 1,37 & 8 & 0,71 & 1,04 \\
\hline Richardia humistrata & Rubiaceae & 40 & 1,37 & 8 & 0,71 & 1,04 \\
\hline Croton $\mathrm{sp}$. & Euphorbiaceae & 20 & 0,69 & 15 & 1,34 & 1,01 \\
\hline Senecio leptolobus & Asteraceae & 30 & 1,03 & 11 & 0,98 & 1,01 \\
\hline Nothoscordum cf. bivalve & Alliaceae & 40 & 1,37 & 4 & 0,36 & 0,87 \\
\hline Desmodium incanum & Fabaceae & 40 & 1,37 & 4 & 0,36 & 0,87 \\
\hline Oxalis eriocarpa & Oxalidaceae & 40 & 1,37 & 4 & 0,36 & 0,87 \\
\hline Setaria parviflora & Poaceae & 40 & 1,37 & 4 & 0,36 & 0,87 \\
\hline Aristida murina & Poaceae & 20 & 0,69 & 11 & 0,98 & 0,84 \\
\hline Stipa setigera & Poaceae & 20 & 0,69 & 11 & 0,98 & 0,84 \\
\hline Facelis retusa & Asteraceae & 30 & 1,03 & 7 & 0,63 & 0,83 \\
\hline Aristida laevis & Poaceae & 30 & 1,03 & 7 & 0,63 & 0,83 \\
\hline Senecio brasiliensis & Asteraceae & 30 & 1,03 & 3 & 0,27 & 0,65 \\
\hline Wahlenbergia linarioides & Campanulaceae & 30 & 1,03 & 3 & 0,27 & 0,65 \\
\hline Herbertia lahue & Iridaceae & 30 & 1,03 & 3 & 0,27 & 0,65 \\
\hline Cliococca selaginoides & Linaceae & 30 & 1,03 & 3 & 0,27 & 0,65 \\
\hline Cuphea glutinosa & Lythraceae & 30 & 1,03 & 3 & 0,27 & 0,65 \\
\hline Polygala australis & Polygalaceae & 30 & 1,03 & 3 & 0,27 & 0,65 \\
\hline Galium richardianum & Rubiaceae & 30 & 1,03 & 3 & 0,27 & 0,65 \\
\hline Polycarpon tetraphyllum & Caryophyllaceae & 20 & 0,69 & 6 & 0,54 & 0,61 \\
\hline Schizachyrium spicatum & Poaceae & 20 & 0,69 & 6 & 0,54 & 0,61 \\
\hline Sporobolus indicus & Poaceae & 20 & 0,69 & 6 & 0,54 & 0,61 \\
\hline Chaptalia mandonii & Asteraceae & 20 & 0,69 & 2 & 0,18 & 0,43 \\
\hline Conyza bonariensis & Asteraceae & 20 & 0,69 & 2 & 0,18 & 0,43 \\
\hline Gamochaeta falcata & Asteraceae & 20 & 0,69 & 2 & 0,18 & 0,43 \\
\hline Soliva sessilis & Asteraceae & 20 & 0,69 & 2 & 0,18 & 0,43 \\
\hline Euphorbia selloi & Euphorbiaceae & 20 & 0,69 & 2 & 0,18 & 0,43 \\
\hline Galactia marginalis & Fabaceae & 20 & 0,69 & 2 & 0,18 & 0,43 \\
\hline
\end{tabular}


Tabela 2A. Continuação

\begin{tabular}{|c|c|c|c|c|c|c|}
\hline Campo rupestre/Táxons & Famílias & FA & FR & $\mathrm{CA}$ & $\mathrm{CR}$ & IVI \\
\hline Stylosanthes montevidensis & Fabaceae & 20 & 0,69 & 2 & 0,18 & 0,43 \\
\hline Zygostigma australe & Gentianaceae & 20 & 0,69 & 2 & 0,18 & 0,43 \\
\hline Oxalis conorrhiza & Oxalidaceae & 20 & 0,69 & 2 & 0,18 & 0,43 \\
\hline Bothriochloa laguroides & Poaceae & 20 & 0,69 & 2 & 0,18 & 0,43 \\
\hline Eragrostis lugens & Poaceae & 20 & 0,69 & 2 & 0,18 & 0,43 \\
\hline Piptochaetium stipoides & Poaceae & 20 & 0,69 & 2 & 0,18 & 0,43 \\
\hline Richardia stellaris & Rubiaceae & 20 & 0,69 & 2 & 0,18 & 0,43 \\
\hline Pfaffia gnaphaloides & Amaranthaceae & 10 & 0,34 & 5 & 0,45 & 0,40 \\
\hline Lessingianthus macrocephalus & Asteraceae & 10 & 0,34 & 5 & 0,45 & 0,40 \\
\hline Parodia erinacea & Cactaceae & 10 & 0,34 & 5 & 0,45 & 0,40 \\
\hline Stylosanthes leiocarpa & Fabaceae & 10 & 0,34 & 5 & 0,45 & 0,40 \\
\hline Elionurus sp. & Poaceae & 10 & 0,34 & 5 & 0,45 & 0,40 \\
\hline Melica rigida & Poaceae & 10 & 0,34 & 5 & 0,45 & 0,40 \\
\hline Microchloa indica & Poaceae & 10 & 0,34 & 5 & 0,45 & 0,40 \\
\hline Galium hirtum & Rubiaceae & 10 & 0,34 & 5 & 0,45 & 0,40 \\
\hline Chenopodium retusum & Amaranthaceae & 10 & 0,34 & 1 & 0,09 & 0,22 \\
\hline Pfaffia tuberosa & Amaranthaceae & 10 & 0,34 & 1 & 0,09 & 0,22 \\
\hline Cyclospermum leptophyllum & Apiaceae & 10 & 0,34 & 1 & 0,09 & 0,22 \\
\hline Eryngium nudicaule & Apiaceae & 10 & 0,34 & 1 & 0,09 & 0,22 \\
\hline Conyza primulifolia & Asteraceae & 10 & 0,34 & 1 & 0,09 & 0,22 \\
\hline Lucilia nitens & Asteraceae & 10 & 0,34 & 1 & 0,09 & 0,22 \\
\hline Paronychia brasiliana & Caryophyllaceae & 10 & 0,34 & 1 & 0,09 & 0,22 \\
\hline Dichondra macrocalyx & Convolvulaceae & 10 & 0,34 & 1 & 0,09 & 0,22 \\
\hline Bulbostylis capillaris fo. stenantha & Cyperaceae & 10 & 0,34 & 1 & 0,09 & 0,22 \\
\hline Bulbostylis juncoides & Cyperaceae & 10 & 0,34 & 1 & 0,09 & 0,22 \\
\hline Cyperus aggregatus & Cyperaceae & 10 & 0,34 & 1 & 0,09 & 0,22 \\
\hline Ditaxis acaulis & Euphorbiaceae & 10 & 0,34 & 1 & 0,09 & 0,22 \\
\hline Pomaria pilosa & Fabaceae & 10 & 0,34 & 1 & 0,09 & 0,22 \\
\hline Trifolium polymorphum & Fabaceae & 10 & 0,34 & 1 & 0,09 & 0,22 \\
\hline Zornia sp. & Fabaceae & 10 & 0,34 & 1 & 0,09 & 0,22 \\
\hline Hyptis mutabilis & Lamiaceae & 10 & 0,34 & 1 & 0,09 & 0,22 \\
\hline Dorstenia brasiliensis & Moraceae & 10 & 0,34 & 1 & 0,09 & 0,22 \\
\hline Oenothera sp. & Onagraceae & 10 & 0,34 & 1 & 0,09 & 0,22 \\
\hline Brachystelle camporum & Orchidaceae & 10 & 0,34 & 1 & 0,09 & 0,22 \\
\hline Turnera sidoides & Passifloraceae & 10 & 0,34 & 1 & 0,09 & 0,22 \\
\hline Briza minor & Poaceae & 10 & 0,34 & 1 & 0,09 & 0,22 \\
\hline Eleusine tristachya & Poaceae & 10 & 0,34 & 1 & 0,09 & 0,22 \\
\hline Piptochaetium lasianthum & Poaceae & 10 & 0,34 & 1 & 0,09 & 0,22 \\
\hline Polygala linoides & Polygalaceae & 10 & 0,34 & 1 & 0,09 & 0,22 \\
\hline Galianthe fastigiata & Rubiaceae & 10 & 0,34 & 1 & 0,09 & 0,22 \\
\hline Solanum hasslerianum & Solanaceae & 10 & 0,34 & 1 & 0,09 & 0,22 \\
\hline Total & & 2910 & 100 & 1119 & 100 & 100 \\
\hline
\end{tabular}

Tabela 2B. Parâmetros quantitativos avaliados no campo seco. $\mathrm{FA}=$ frequência absoluta; $\mathrm{FR}=$ frequência relativa; $\mathrm{CA}=$ cobertura absoluta; $\mathrm{CR}=$ cobertura relativa e IVI = índice de valor de importância.

\begin{tabular}{|c|c|c|c|c|c|c|}
\hline Campo seco/Táxons & Famílias & FA & FR & $\mathrm{CA}$ & $\mathrm{CR}$ & IVI \\
\hline Paspalum notatum & Poaceae & 90 & 2,85 & 196 & 14,79 & 8,82 \\
\hline Vernonanthura nudiflora & Asteraceae & 80 & 2,53 & 145 & 10,94 & 6,74 \\
\hline Vulpia bromoides & Poaceae & 80 & 2,53 & 88 & 6,64 & 4,59 \\
\hline Mnesithea selloana & Poaceae & 90 & 2,85 & 64 & 4,83 & 3,84 \\
\hline Eryngium horridum & Apiaceae & 80 & 2,53 & 65 & 4,91 & 3,72 \\
\hline Piptochaetium montevidense & Poaceae & 70 & 2,22 & 61 & 4,60 & 3,41 \\
\hline Acanthostyles buniifolius & Asteraceae & 60 & 1,90 & 48 & 3,62 & 2,76 \\
\hline
\end{tabular}


Tabela 2B. Continuação

\begin{tabular}{|c|c|c|c|c|c|c|}
\hline Campo seco/Táxons & Famílias & FA & FR & $\mathrm{CA}$ & CR & IVI \\
\hline Stipa setigera & Poaceae & 70 & 2,22 & 41 & 3,09 & 2,65 \\
\hline Baccharis crispa & Asteraceae & 40 & 1,27 & 51 & 3,85 & 2,56 \\
\hline Desmodium incanum & Fabaceae & 80 & 2,53 & 25 & 1,89 & 2,21 \\
\hline Hypochaeris neopinnatifida & Asteraceae & 70 & 2,22 & 24 & 1,81 & 2,01 \\
\hline Axonopus affinis & Poaceae & 50 & 1,58 & 32 & 2,42 & 2,00 \\
\hline Chascolytrum subaristatum & Poaceae & 70 & 2,22 & 20 & 1,51 & 1,86 \\
\hline Steinchisma hians & Poaceae & 70 & 2,22 & 19 & 1,43 & 1,82 \\
\hline Dichondra sericea & Convolvulaceae & 60 & 1,90 & 22 & 1,66 & 1,78 \\
\hline Paspalum plicatulum & Poaceae & 30 & 0,95 & 32 & 2,42 & 1,68 \\
\hline Wahlenbergia linarioides & Campanulaceae & 80 & 2,53 & 8 & 0,60 & 1,57 \\
\hline Hyptis mutabilis & Lamiaceae & 70 & 2,22 & 11 & 0,83 & 1,52 \\
\hline Setaria parviflora & Poaceae & 70 & 2,22 & 11 & 0,83 & 1,52 \\
\hline Trifolium polymorphum & Fabaceae & 70 & 2,22 & 11 & 0,83 & 1,52 \\
\hline Melica rigida & Poaceae & 60 & 1,90 & 15 & 1,13 & 1,52 \\
\hline Galium richardianum & Rubiaceae & 70 & 2,22 & 7 & 0,53 & 1,37 \\
\hline Eryngium nudicaule & Apiaceae & 20 & 0,63 & 25 & 1,89 & 1,26 \\
\hline Piptochaetium lasianthum & Poaceae & 30 & 0,95 & 20 & 1,51 & 1,23 \\
\hline Conyza bonariensis & Asteraceae & 60 & 1,90 & 6 & 0,45 & 1,18 \\
\hline Andropogon ternatus & Poaceae & 50 & 1,58 & 9 & 0,68 & 1,13 \\
\hline Schizachyrium microstachyum & Poaceae & 40 & 1,27 & 12 & 0,91 & 1,09 \\
\hline Baccharis coridifolia & Asteraceae & 20 & 0,63 & 20 & 1,51 & 1,07 \\
\hline Senecio brasiliensis & Asteraceae & 50 & 1,58 & 5 & 0,38 & 0,98 \\
\hline Sisyrinchium micranthum & Iridaceae & 50 & 1,58 & 5 & 0,38 & 0,98 \\
\hline Richardia humistrata & Rubiaceae & 50 & 1,58 & 5 & 0,38 & 0,98 \\
\hline Stenachaenium campestre & Asteraceae & 30 & 0,95 & 11 & 0,83 & 0,89 \\
\hline Cyclospermum leptophyllum & Apiaceae & 40 & 1,27 & 4 & 0,30 & 0,78 \\
\hline Evolvulus sericeus & Convovulaceae & 40 & 1,27 & 4 & 0,30 & 0,78 \\
\hline Commelina platyphylla & Commelinaceae & 40 & 1,27 & 4 & 0,30 & 0,78 \\
\hline Hypoxis decumbens & Hypoxidaceae & 40 & 1,27 & 4 & 0,30 & 0,78 \\
\hline Borreria dasycephala & Rubiaceae & 40 & 1,27 & 4 & 0,30 & 0,78 \\
\hline Senecio selloi & Asteraceae & 30 & 0,95 & 7 & 0,53 & 0,74 \\
\hline Oxalis eriocarpa & Oxalidaceae & 30 & 0,95 & 7 & 0,53 & 0,74 \\
\hline Oxalis articulata & Oxalidaceae & 20 & 0,63 & 10 & 0,75 & 0,69 \\
\hline Chaptalia mandonii & Asteraceae & 30 & 0,95 & 3 & 0,23 & 0,59 \\
\hline Facelis retusa & Asteraceae & 30 & 0,95 & 3 & 0,23 & 0,59 \\
\hline Pterocaulon sp. 1 & Asteraceae & 30 & 0,95 & 3 & 0,23 & 0,59 \\
\hline Soliva sessilis & Asteraceae & 30 & 0,95 & 3 & 0,23 & 0,59 \\
\hline Euphorbia selloi & Euphorbiaceae & 30 & 0,95 & 3 & 0,23 & 0,59 \\
\hline Herbertia lahue & Iridaceae & 30 & 0,95 & 3 & 0,23 & 0,59 \\
\hline Aristida venustula & Poaceae & 30 & 0,95 & 3 & 0,23 & 0,59 \\
\hline Briza minor & Poaceae & 30 & 0,95 & 3 & 0,23 & 0,59 \\
\hline Carex sororia & Cyperaceae & 20 & 0,63 & 6 & 0,45 & 0,54 \\
\hline Rhynchosia diversifolia & Fabaceae & 20 & 0,63 & 6 & 0,45 & 0,54 \\
\hline Oenothera sp. & Onagraceae & 20 & 0,63 & 6 & 0,45 & 0,54 \\
\hline Bothriochloa laguroides & Poaceae & 20 & 0,63 & 6 & 0,45 & 0,54 \\
\hline Danthonia montevidensis & Poaceae & 20 & 0,63 & 6 & 0,45 & 0,54 \\
\hline Piptochaetium stipoides & Poaceae & 20 & 0,63 & 6 & 0,45 & 0,54 \\
\hline Galianthe fastigiata & Rubiaceae & 20 & 0,63 & 6 & 0,45 & 0,54 \\
\hline
\end{tabular}


Tabela 2B. Continuação

\begin{tabular}{|c|c|c|c|c|c|c|}
\hline Campo seco/Táxons & Famílias & FA & FR & $\mathrm{CA}$ & $\mathrm{CR}$ & IVI \\
\hline Aristida jubata & Poaceae & 10 & 0,32 & 10 & 0,75 & 0,54 \\
\hline Aristida laevis & Poaceae & 10 & 0,32 & 10 & 0,75 & 0,54 \\
\hline Pfaffia tuberosa & Amaranthaceae & 20 & 0,63 & 2 & 0,15 & 0,39 \\
\hline Chrysolaena flexuosa & Asteraceae & 20 & 0,63 & 2 & 0,15 & 0,39 \\
\hline Conyza floribunda & Asteraceae & 20 & 0,63 & 2 & 0,15 & 0,39 \\
\hline Carex phalaroides & Cyperaceae & 20 & 0,63 & 2 & 0,15 & 0,39 \\
\hline Kyllinga odorata & Cyperaceae & 20 & 0,63 & 2 & 0,15 & 0,39 \\
\hline Kelissa brasiliensis & Iridaceae & 20 & 0,63 & 2 & 0,15 & 0,39 \\
\hline Cuphea glutinosa & Lythraceae & 20 & 0,63 & 2 & 0,15 & 0,39 \\
\hline Oxalis brasiliensis & Oxalidaceae & 20 & 0,63 & 2 & 0,15 & 0,39 \\
\hline Plantago tomentosa & Plantaginaceae & 20 & 0,63 & 2 & 0,15 & 0,39 \\
\hline Axonopus suffultus & Poaceae & 20 & 0,63 & 2 & 0,15 & 0,39 \\
\hline Dichanthelium sabulorum & Poaceae & 20 & 0,63 & 2 & 0,15 & 0,39 \\
\hline Lolium multiflorum & Poaceae & 20 & 0,63 & 2 & 0,15 & 0,39 \\
\hline Hypochaeris chillensis & Asteraceae & 10 & 0,32 & 5 & 0,38 & 0,35 \\
\hline Lessingianthus sellowii & Asteraceae & 10 & 0,32 & 5 & 0,38 & 0,35 \\
\hline Mimosa flagellaris & Fabaceae & 10 & 0,32 & 5 & 0,38 & 0,35 \\
\hline Cliococca selaginoides & Linaceae & 10 & 0,32 & 5 & 0,38 & 0,35 \\
\hline Nierembergia cf. riograndensis & Solanaceae & 10 & 0,32 & 5 & 0,38 & 0,35 \\
\hline Aloysia lycioides & Verbenaceae & 10 & 0,32 & 5 & 0,38 & 0,35 \\
\hline Nothoscordum cf. bivalve & Alliaceae & 10 & 0,32 & 1 & 0,08 & 0,20 \\
\hline Hydrocotyle exigua & Araliaceae & 10 & 0,32 & 1 & 0,08 & 0,20 \\
\hline Ambrosia elatior & Asteraceae & 10 & 0,32 & 1 & 0,08 & 0,20 \\
\hline Gamochaeta coarctata & Asteraceae & 10 & 0,32 & 1 & 0,08 & 0,20 \\
\hline Hypochaeris albiflora & Asteraceae & 10 & 0,32 & 1 & 0,08 & 0,20 \\
\hline Hypochaeris glabra & Asteraceae & 10 & 0,32 & 1 & 0,08 & 0,20 \\
\hline Orthopappus angustifolius & Asteraceae & 10 & 0,32 & 1 & 0,08 & 0,20 \\
\hline Triodanis perfoliata subsp. biflora & Campanulaceae & 10 & 0,32 & 1 & 0,08 & 0,20 \\
\hline Cerastium glomeratum & Caryophyllaceae & 10 & 0,32 & 1 & 0,08 & 0,20 \\
\hline Abildgaardia ovata & Cyperaceae & 10 & 0,32 & 1 & 0,08 & 0,20 \\
\hline Bulbostylis capillaris fo. stenantha & Cyperaceae & 10 & 0,32 & 1 & 0,08 & 0,20 \\
\hline Bulbostylis juncoides & Cyperaceae & 10 & 0,32 & 1 & 0,08 & 0,20 \\
\hline Cyperus reflexus & Cyperaceae & 10 & 0,32 & 1 & 0,08 & 0,20 \\
\hline Galactia marginalis & Fabaceae & 10 & 0,32 & 1 & 0,08 & 0,20 \\
\hline Sisyrinchium megapotamicum & Iridaceae & 10 & 0,32 & 1 & 0,08 & 0,20 \\
\hline Juncus capillaceus & Juncaceae & 10 & 0,32 & 1 & 0,08 & 0,20 \\
\hline Sida rhombifolia & Malvaceae & 10 & 0,32 & 1 & 0,08 & 0,20 \\
\hline Oxalis conorrhiza & Oxalidaceae & 10 & 0,32 & 1 & 0,08 & 0,20 \\
\hline Oxalis lasiopetala & Oxalidaceae & 10 & 0,32 & 1 & 0,08 & 0,20 \\
\hline Eleusine tristachya & Poaceae & 10 & 0,32 & 1 & 0,08 & 0,20 \\
\hline Eragrostis neesii & Poaceae & 10 & 0,32 & 1 & 0,08 & 0,20 \\
\hline Paspalum lepton & Poaceae & 10 & 0,32 & 1 & 0,08 & 0,20 \\
\hline Paspalum maculosum & Poaceae & 10 & 0,32 & 1 & 0,08 & 0,20 \\
\hline Sporobolus indicus & Poaceae & 10 & 0,32 & 1 & 0,08 & 0,20 \\
\hline Borreria verticillata & Rubiaceae & 10 & 0,32 & 1 & 0,08 & 0,20 \\
\hline Richardia stellaris & Rubiaceae & 10 & 0,32 & 1 & 0,08 & 0,20 \\
\hline Verbena montevidensis & Verbenaceae & 10 & 0,32 & 1 & 0,08 & 0,20 \\
\hline Total & & 3160 & 100 & 1325 & 100 & 100 \\
\hline
\end{tabular}


Tabela 2C. Parâmetros quantitativos avaliados no campo úmido. $\mathrm{FA}=$ frequência absoluta; $\mathrm{FR}=$ frequência relativa; $\mathrm{CA}=$ cobertura absoluta; $\mathrm{CR}$ = cobertura relativa e IVI = índice de valor de importância.

\begin{tabular}{|c|c|c|c|c|c|c|}
\hline Campo úmido/Táxons & Famílias & FA & FR & $\mathrm{CA}$ & $\mathrm{CR}$ & IVI \\
\hline Axonopus affinis & Poaceae & 90 & 5,33 & 172 & 14,68 & 10,00 \\
\hline Paspalum pumilum & Poaceae & 70 & 4,14 & 171 & 14,59 & 9,37 \\
\hline Eleocharis bonariensis & Cyperaceae & 70 & 4,14 & 170 & 14,51 & 9,32 \\
\hline Eleocharis viridans & Cyperaceae & 70 & 4,14 & 150 & 12,80 & 8,47 \\
\hline Luziola peruviana & Poaceae & 70 & 4,14 & 61 & 5,20 & 4,67 \\
\hline Paspalum dilatatum & Poaceae & 70 & 4,14 & 52 & 4,44 & 4,29 \\
\hline Hydrocotyle ranunculoides & Araliaceae & 60 & 3,55 & 54 & 4,61 & 4,08 \\
\hline Leersia hexandra & Poaceae & 70 & 4,14 & 32 & 2,73 & 3,44 \\
\hline Sisyrinchium micranthum & Iridaceae & 80 & 4,73 & 16 & 1,37 & 3,05 \\
\hline Bacopa sp. & Plantaginaceae & 70 & 4,14 & 19 & 1,62 & 2,88 \\
\hline Poa annua & Poaceae & 60 & 3,55 & 18 & 1,54 & 2,54 \\
\hline Eleocharis sellowiana & Cyperaceae & 30 & 1,78 & 32 & 2,73 & 2,25 \\
\hline Lobelia hederacea & Campanulaceae & 40 & 2,37 & 22 & 1,88 & 2,12 \\
\hline Crassula longipes & Crassulaceae & 60 & 3,55 & 6 & 0,51 & 2,03 \\
\hline Lilaeopsis brasiliensis & Apiaceae & 30 & 1,78 & 20 & 1,71 & 1,74 \\
\hline Paspalum lepton & Poaceae & 20 & 1,18 & 25 & 2,13 & 1,66 \\
\hline Ranunculus bonariensis & Ranunculaceae & 40 & 2,37 & 8 & 0,68 & 1,52 \\
\hline Kyllinga brevifolia & Cyperaceae & 30 & 1,78 & 11 & 0,94 & 1,36 \\
\hline Steinchisma decipiens & Poaceae & 40 & 2,37 & 4 & 0,34 & 1,35 \\
\hline Gamochaeta purpurea & Asteraceae & 30 & 1,78 & 7 & 0,60 & 1,19 \\
\hline Senecio heterotrichius & Asteraceae & 30 & 1,78 & 7 & 0,60 & 1,19 \\
\hline Juncus tenuis & Juncaceae & 30 & 1,78 & 7 & 0,60 & 1,19 \\
\hline Chascolytrum subaristatum & Poaceae & 30 & 1,78 & 7 & 0,60 & 1,19 \\
\hline Paspalum vaginatum & Poaceae & 10 & 0,59 & 20 & 1,71 & 1,15 \\
\hline Paspalum notatum & Poaceae & 20 & 1,18 & 10 & 0,85 & 1,02 \\
\hline Gamochaeta coarctata & Asteraceae & 30 & 1,78 & 3 & 0,26 & 1,02 \\
\hline Plantago tomentosa & Plantaginaceae & 30 & 1,78 & 3 & 0,26 & 1,02 \\
\hline Juncus microcephalus & Juncaceae & 20 & 1,18 & 6 & 0,51 & 0,85 \\
\hline Chascolytrum poomorphum & Poaceae & 20 & 1,18 & 6 & 0,51 & 0,85 \\
\hline Lolium multiflorum & Poaceae & 20 & 1,18 & 6 & 0,51 & 0,85 \\
\hline Paspalum plicatulum & Poaceae & 20 & 1,18 & 6 & 0,51 & 0,85 \\
\hline Cyclospermum leptophyllum & Apiaceae & 20 & 1,18 & 2 & 0,17 & 0,68 \\
\hline Baccharis crispa & Asteraceae & 20 & 1,18 & 2 & 0,17 & 0,68 \\
\hline Senecio brasiliensis & Asteraceae & 20 & 1,18 & 2 & 0,17 & 0,68 \\
\hline Soliva sessilis & Asteraceae & 20 & 1,18 & 2 & 0,17 & 0,68 \\
\hline Cerastium $\mathrm{sp}$. & Caryophyllaceae & 20 & 1,18 & 2 & 0,17 & 0,68 \\
\hline Kyllinga odorata & Cyperaceae & 20 & 1,18 & 2 & 0,17 & 0,68 \\
\hline Juncus capillaceus & Juncaceae & 20 & 1,18 & 2 & 0,17 & 0,68 \\
\hline Andropogon lateralis & Poaceae & 20 & 1,18 & 2 & 0,17 & 0,68 \\
\hline Hydrocotyle bonariensis & Araliaceae & 10 & 0,59 & 5 & 0,43 & 0,51 \\
\hline Cyperus rigens & Cyperaceae & 10 & 0,59 & 5 & 0,43 & 0,51 \\
\hline Dichondra sericea & Convolvulaceae & 10 & 0,59 & 1 & 0,09 & 0,34 \\
\hline Carex bonariensis & Cyperaceae & 10 & 0,59 & 1 & 0,09 & 0,34 \\
\hline Trifolium polymorphum & Fabaceae & 10 & 0,59 & 1 & 0,09 & 0,34 \\
\hline Scutellaria racemosa & Lamiaceae & 10 & 0,59 & 1 & 0,09 & 0,34 \\
\hline Cuphea glutinosa & Lythraceae & 10 & 0,59 & 1 & 0,09 & 0,34 \\
\hline Oxalis brasiliensis & Oxalidaceae & 10 & 0,59 & 1 & 0,09 & 0,34 \\
\hline Bothriochloa laguroides & Poaceae & 10 & 0,59 & 1 & 0,09 & 0,34 \\
\hline Chascolytrum rufum & Poaceae & 10 & 0,59 & 1 & 0,09 & 0,34 \\
\hline Panicum schwackeanum & Poaceae & 10 & 0,59 & 1 & 0,09 & 0,34 \\
\hline Phalaris angusta & Poaceae & 10 & 0,59 & 1 & 0,09 & 0,34 \\
\hline Sporobolus indicus & Poaceae & 10 & 0,59 & 1 & 0,09 & 0,34 \\
\hline Polygonum punctatum & Polygonaceae & 10 & 0,59 & 1 & 0,09 & 0,34 \\
\hline Galium hirtum & Rubiaceae & 10 & 0,59 & 1 & 0,09 & 0,34 \\
\hline Scrophularia peregrina & Schrophulariaceae & 10 & 0,59 & 1 & 0,09 & 0,34 \\
\hline Verbena montevidensis & Verbenaceae & 10 & 0,59 & 1 & 0,09 & 0,34 \\
\hline Total & & 1690 & 100 & 1172 & 100 & 100 \\
\hline
\end{tabular}


Tabela 2D. Parâmetros quantitativos avaliados no campo brejoso. FA = frequência absoluta; FR = frequência relativa; CA = cobertura absoluta; CR $=$ cobertura relativa e IVI = índice de valor de importância.

\begin{tabular}{|c|c|c|c|c|c|c|}
\hline Campo brejoso/Táxons & Famílias & FA & FR & $\mathrm{CA}$ & $\mathrm{CR}$ & IVI \\
\hline Eleocharis viridans & Cyperaceae & 50 & 3,23 & 161 & 15,15 & 9,19 \\
\hline Rhynchospora conferta & Cyperaceae & 70 & 4,52 & 111 & 10,44 & 7,48 \\
\hline Rhynchospora marisculus & Cyperaceae & 80 & 5,16 & 47 & 4,42 & 4,79 \\
\hline Andropogon lateralis & Poaceae & 50 & 3,23 & 65 & 6,11 & 4,67 \\
\hline Hygrophila costata & Acanthaceae & 80 & 5,16 & 41 & 3,86 & 4,51 \\
\hline Chascolytrum uniolae & Poaceae & 60 & 3,87 & 50 & 4,70 & 4,29 \\
\hline Rhynchospora scutellata & Cyperaceae & 50 & 3,23 & 56 & 5,27 & 4,25 \\
\hline Baccharis crispa & Asteraceae & 60 & 3,87 & 47 & 4,42 & 4,15 \\
\hline Paspalum dilatatum & Poaceae & 50 & 3,23 & 45 & 4,23 & 3,73 \\
\hline Luziola peruviana & Poaceae & 50 & 3,23 & 42 & 3,95 & 3,59 \\
\hline Echinodorus grandiflorus & Alismataceae & 30 & 1,94 & 51 & 4,80 & 3,37 \\
\hline Paspalum pumilum & Poaceae & 40 & 2,58 & 43 & 4,05 & 3,31 \\
\hline Bacopa sp. & Plantaginaceae & 70 & 4,52 & 20 & 1,88 & 3,20 \\
\hline Andropogon virgatus & Poaceae & 40 & 2,58 & 36 & 3,39 & 2,98 \\
\hline Eleocharis bonariensis & Cyperaceae & 30 & 1,94 & 36 & 3,39 & 2,66 \\
\hline Lilaeopsis brasiliensis & Apiaceae & 60 & 3,87 & 14 & 1,32 & 2,59 \\
\hline Lobelia hederacea & Campanulaceae & 50 & 3,23 & 18 & 1,69 & 2,46 \\
\hline Axonopus affinis & Poaceae & 50 & 3,23 & 18 & 1,69 & 2,46 \\
\hline Paspalum exaltatum & Poaceae & 20 & 1,29 & 35 & 3,29 & 2,29 \\
\hline Hydrocotyle ranunculoides & Araliaceae & 30 & 1,94 & 16 & 1,51 & 1,72 \\
\hline Saccharum villosum & Poaceae & 30 & 1,94 & 16 & 1,51 & 1,72 \\
\hline Gamochaeta coarctata & Asteraceae & 30 & 1,94 & 7 & 0,66 & 1,30 \\
\hline Senecio heterotrichius & Asteraceae & 30 & 1,94 & 7 & 0,66 & 1,30 \\
\hline Chascolytrum poomorphum & Poaceae & 30 & 1,94 & 7 & 0,66 & 1,30 \\
\hline Thelypteris sp. & Thelypteridaceae & 30 & 1,94 & 7 & 0,66 & 1,30 \\
\hline Pycreus megapotamicus & Cyperaceae & 20 & 1,29 & 11 & 1,03 & 1,16 \\
\hline Eleocharis sellowiana & Cyperaceae & 20 & 1,29 & 10 & 0,94 & 1,12 \\
\hline Achyrocline alata & Asteraceae & 30 & 1,94 & 3 & 0,28 & 1,11 \\
\hline Cerastium rivulare & Caryophyllaceae & 20 & 1,29 & 6 & 0,56 & 0,93 \\
\hline Leersia hexandra & Poaceae & 20 & 1,29 & 6 & 0,56 & 0,93 \\
\hline Xyris sp. & Xyridaceae & 20 & 1,29 & 6 & 0,56 & 0,93 \\
\hline Hypochaeris lutea & Asteraceae & 20 & 1,29 & 2 & 0,19 & 0,74 \\
\hline Pterocaulon cf. balansae & Asteraceae & 20 & 1,29 & 2 & 0,19 & 0,74 \\
\hline Galium hirtum & Rubiaceae & 20 & 1,29 & 2 & 0,19 & 0,74 \\
\hline Xyris jupicai & Xyridaceae & 20 & 1,29 & 2 & 0,19 & 0,74 \\
\hline Eryngium pandanifolium & Apiaceae & 10 & 0,65 & 1 & 0,09 & 0,37 \\
\hline Baccharis dracunculifolia & Asteraceae & 10 & 0,65 & 1 & 0,09 & 0,37 \\
\hline Baccharis junciformis & Asteraceae & 10 & 0,65 & 1 & 0,09 & 0,37 \\
\hline Chaptalia runcinata & Asteraceae & 10 & 0,65 & 1 & 0,09 & 0,37 \\
\hline Conyza bonariensis & Asteraceae & 10 & 0,65 & 1 & 0,09 & 0,37 \\
\hline Pterocaulon sp. 2 & Asteraceae & 10 & 0,65 & 1 & 0,09 & 0,37 \\
\hline Senecio brasiliensis & Asteraceae & 10 & 0,65 & 1 & 0,09 & 0,37 \\
\hline Senecio icoglossus & Asteraceae & 10 & 0,65 & 1 & 0,09 & 0,37 \\
\hline Cerastium commersonianum & Caryophyllaceae & 10 & 0,65 & 1 & 0,09 & 0,37 \\
\hline Carex longii & Cyperaceae & 10 & 0,65 & 1 & 0,09 & 0,37 \\
\hline Hypoxis decumbens & Hipoxidaceae & 10 & 0,65 & 1 & 0,09 & 0,37 \\
\hline Sisyrinchium platense & Iridaceae & 10 & 0,65 & 1 & 0,09 & 0,37 \\
\hline Juncus microcephalus & Juncaceae & 10 & 0,65 & 1 & 0,09 & 0,37 \\
\hline Scutellaria racemosa & Lamiaceae & 10 & 0,65 & 1 & 0,09 & 0,37 \\
\hline Tibouchina gracilis & Melastomataceae & 10 & 0,65 & 1 & 0,09 & 0,37 \\
\hline Andropogon ternatus & Poaceae & 10 & 0,65 & 1 & 0,09 & 0,37 \\
\hline Polygonum punctatum & Polygalaceae & 10 & 0,65 & 1 & 0,09 & 0,37 \\
\hline Total & & 1550 & 100 & 1063 & 100 & 100 \\
\hline
\end{tabular}




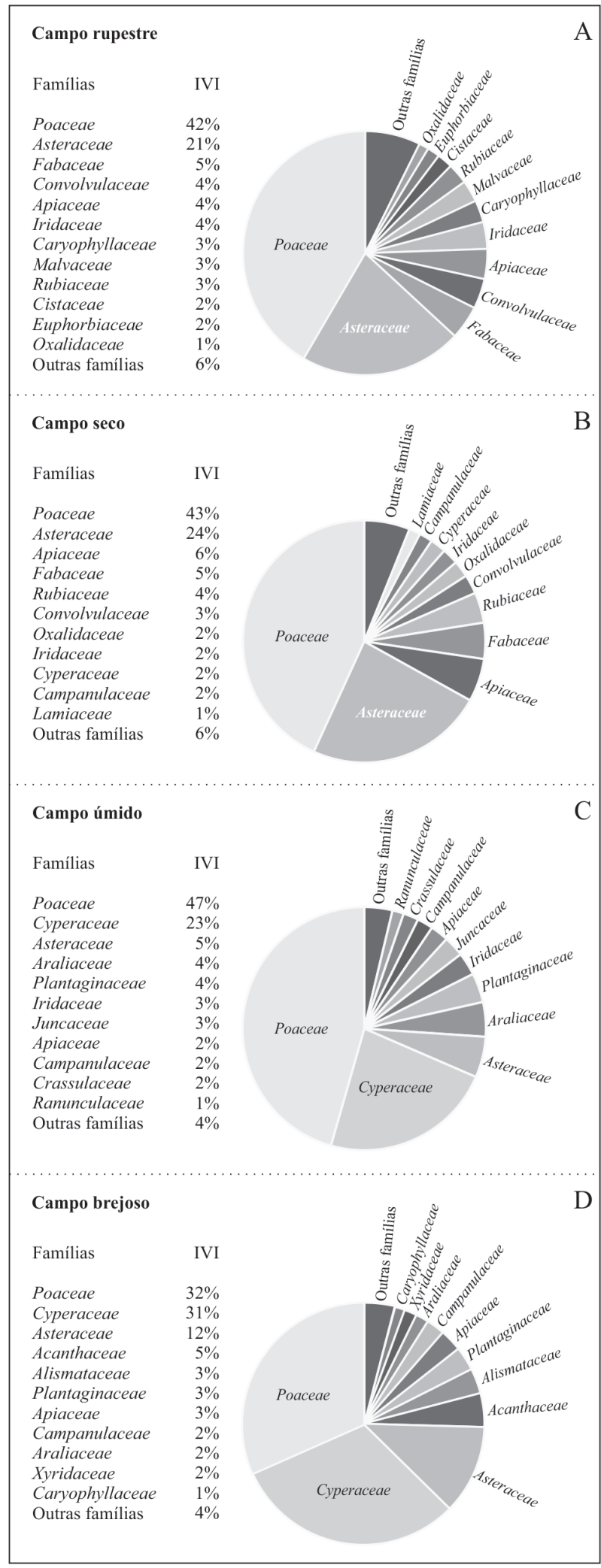

Figs. 4A-D. Diagramas de porcentagem mostrando o somatório dos IVI por família em cada fitofisionomia, considerando-se todas as espécies registradas nas UAs. A. Campo rupestre; B. Campo seco; C. Campo úmido; D. Campo brejoso
Leersia hexandra $(-0,51)$ e Lilaeopsis brasiliensis $(-0,50)$. O eixo 2 da ordenação apresentou uma variância de $8,93 \%$. A porção positiva do eixo 2 agregou as UAs de campo seco e úmido e, a porção negativa, uniu as UAs de campo rupestre e brejoso. As espécies com maior coeficiente de correlação foram registradas somente na porção negativa do segundo eixo, a saber: Chascolytrum uniolae $(-0,63)$, Rhynchospora marisculus $(-0,63)$, Achyrocline alata $(-0,59)$, Andropogon virgatus $(-0,56)$, Andropogon lateralis $(-0,55)$ e Rhynchospora conferta $(-0,54)$.

A separação das UAs na porção positiva e negativa eixo 1 da ordenação sugere uma diferenciação da composição florística quanto à disponibilidade hídrica, onde do lado positivo do eixo permaneceram as UAs associadas a solos bem drenados, e do lado negativo, as UAs em condições de retenção de umidade. No segundo eixo da ordenação, a porção positiva agrupou as comunidades de campo seco e úmido, possivelmente por estas compartilharem características edáficas semelhantes, com solos rasos arenoargilo e argilo-arenoso nas encostas côncavas e convexas, respectivamente (Fig. 1C). A porção negativa do eixo 2 agrupou as comunidades de campo rupestre e brejoso, onde condições extremas xerófitas e hidrófilas prevalecem. No campo rupestre há escassa disponibilidade hídrica, com solos muito rasos ou litossolos (afloramentos graníticos), além de poucos nutrientes. Já no campo brejoso, há grande saturação de água e substratos ligeiramente ácidos, constituídos essencialmente de lama e matéria orgânica decomposta. No eixo 2, nota-se também a separação indubitável dos campos brejoso e úmido, ao passo que, algumas UAs dos campos rupestre e seco se mesclaram, possivelmente devido ao compartilhamento de muitas espécies. Todavia, as UAs dos campos rupestre e seco se concentraram predominantemente em lados opostos do eixo 2. Portanto, a análise exploratória multivariada de PCoA comprova que as quatro fitofisionomias definidas a priori constituem comunidades florísticas heterogêneas.

Este estudo elucidou a riqueza e a diversidade dos Campos arbustivos em São Gabriel. A relevância de ações que visem à proteção dos remanescentes de Campos arbustivos fica ainda mais evidenciada pelo registro de espécies presentes da lista da flora ameaçada de extinção do estado do Rio Grande do Sul e pelo alto grau de conservação da área de estudo, em uma região onde as taxas de conversão do uso do solo estão entre as maiores do Estado.

\section{AGRADECIMENTOS}

Os autores agradecem a Fundação de Amparo à Pesquisa do Estado do Rio Grande do Sul, pelo apoio financeiro concedido através do projeto de pesquisa processo 10121/19; ao Conselho Nacional de Desenvolvimento Científico e Tecnológico, pelo suporte financeiro prestado ao segundo autor, através do auxílio de taxa de bancada processo 141193/2010-7; ao Dr. Guilherme Seger e Bióloga Rosângela Rolim pela concessão das fotos de Porophyllum linifolium (Fig. 3D) e Pfaffia gnaphaloides (Fig. 3I), 


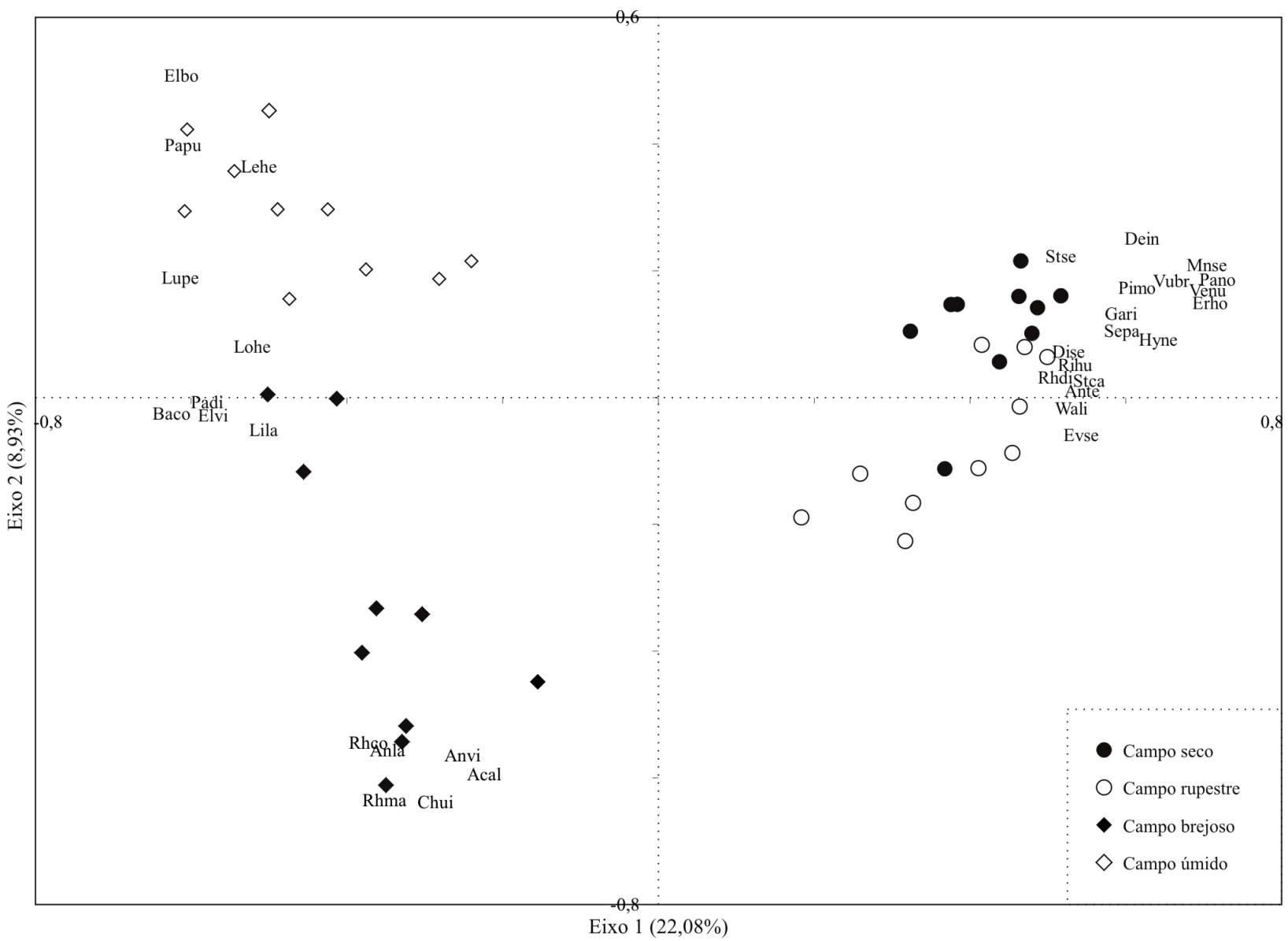

Fig. 5. Diagrama "biplot" de PCoA mostrando a dispersão espacial das UAs e dos táxons que apresentaram maiores valores de correlação. Acal $=$ Achyrocline alata $;$ Anla $=$ Andropogon lateralis $;$ Ante $=$ Andropogon ternatus $; \mathbf{A n v i}=$ Andropogon virgatus $; \mathbf{B a c o}=$ Bacopa $\mathrm{sp} . ; \mathbf{C h u i}=$ Chascolytrum uniolae $;$ Dein $=$ Desmodium incanum $;$ Dise $=$ Dichondra sericea $; \mathbf{E l b o}=$ Eleocharis bonariensis $; \mathbf{E l v i}=$ Eleocharis viridans $; \mathbf{E} \mathbf{r h o}$ $=$ Eryngium horridum $;$ Evse $=$ Evolvulus sericeus $; \mathbf{G a r i}=$ Galium richardianum; Hyne $=$ Hypochaeris neopinnatifida $;$ Lehe $=$ Leersia hexandra $;$ Lila $=$ Lilaeopsis brasiliensis $;$ Lohe $=$ Lobelia hederacea $;$ Lupe $=$ Luziola peruviana $;$ Mnse $=$ Mnesithea selloana $;$ Padi $=$ Paspalum dilatatum; $\mathbf{P a n o}=$ Paspalum notatum $; \mathbf{P a p u}=$ Paspalum pumilum $; \mathbf{P i m o}=$ Piptochaetium montevidense $; \mathbf{R h c o}=$ Rhynchospora conferta $; \mathbf{R h d i}=$ Rhynchosia diversifolia $; \mathbf{R h m a}=$ Rhynchospora marisculus $; \mathbf{R i h u}=$ Richardia humistrata $;$ Sepa $=$ Setaria parviflora $;$ Stca $=$ Stenachaenium campestre $;$ Stse $=$ Stipa setigera $; \mathbf{V e n u}=$ Vernonanthura nudiflora $; \mathbf{V u b r}=$ Vulpia bromoides; $\mathbf{W a l i}=$ Wahlenbergia linarioides.

respectivamente; a colaboração da Dra. Ilsi Iob Boldrini, Dr. Sérgio Augusto de Loreto Bordignon e ao MSc. João Felisberto Larocca e Silva, pelo amparo nas determinações taxonômicas de algumas espécies; ao Dr. Gerhard Ernest Overbeck, pelas sugestões no procedimento analítico. Agradecemos também ao MSc. Paulo Jolar Pazzini Galarça, pelo serviço de ilustração da figura $1 \mathrm{C}$, bem como ao Dr. Paulo Alves de Souza e ao Dr. Aldo Raúl Prieto, pela revisão descritiva dos aspectos fisiográficos da área.

\section{REFERÊNCIAS}

Ab'Sáber, A. 2005. Os domínios de natureza no Brasil: potencialidades paisagísticas. Ateliê, São Paulo. 160p.

Alvares, C.A., Stape, J.L., Sentelhas, P.C., Gonçalves, J.L. de M. \& Sparovek, G. 2013. Köppen's climate classification map for Brazil. Meteorologische Zeitschrift 22(6):711-728.

Andrade, B.O., Koch, C., Boldrini, I.I., Vélez-Martin, E., Hasenack, H., Hermann, J.M., Kollmann, J., Pillar, V.P. \& Overbeck, G.E. 2015. Grassland degradation and restoration: a conceptual framework of stages and thresholds illustrated by southern Brazilian grasslands. Natureza \& Conservação 13:95-104.
Angiosperm Phylogeny Group. APG. 2009. An update of the Angiosperm Phylogeny Group classification for the orders and families of flowering plants: APG III. Botanical Journal of the Linnean Society 161:105-121.

Bilenca, D. \& Miñarro, F. 2004. Identificación de áreas valiosas de pastizal (AVPs) em las Pampas y Campos de Argentina, Uruguay y sur de Brasil. Fundación Vida Silvestre Argentina, Buenos Aires. 323p.

Boldrini, I.I. 2009. A flora dos Campos do Rio Grande do Sul. In Campos Sulinos, conservação e uso sustentável da biodiversidade (V.P. Pillar, S.C. Müller, \& Z.M.S. de Castilhos, eds). Ministério do Meio Ambiente, Brasília, p. 63-77.

Boldrini, I.I., Ferreira, P.M. de A., Andrade, B.O., Schneider, A.A., Setubal, R.B., Trevisan, R. \& Freitas, E.M. 2010. Bioma Pampa: diversidade florística e fisionômica. Pallotti, Porto Alegre. 64p.

Boldrini, I.I., Miotto, S.T.S., Longhi-Wagner, H.M., Pillar,V.P. \& Marzall, K. 1998. Vegetação campestre do Morro da Polícia, Porto Alegre, RS. Acta Botanica Brasilica 12(1):95-106.

Braun-Blanquet, J. 1979. Fitosociologia: bases para el estudio de las comunidades vegetales. H. Blume Ediciones, Madrid. 820p.

Burkart, A. 1975. Evolution of grasses and grasslands in South America. Taxon 24(1):53-66.

Caporal, F.J.M. \& Boldrini, I.I. 2007. Florística e fitossociologia de um campo manejado na Serra do Sudeste, Rio Grande do Sul. Revista Brasileira de Biociências 5(2-3):37-44. 
Constanza, R., d'Arge, R., de Groot, R., Faber, S., Grasso, M., Hannon, B., Limburg, K. Naeen, S. O’Neill, R.V., Paruelo, J., Raskin, R.G., Sutton, P. \& Van den Belt, M. 1997. The value of the world's ecosystem service and natural capital. Nature 387:253-260.

Cordeiro, J.L.P. \& Hasenack, H. 2009. Cobertura vegetal atual do Rio Grande do Sul. In Campos Sulinos, conservação e uso sustentável da biodiversidade (V.P. Pillar, S.C. Müller, \& Z.M.S. de Castilhos, eds). Ministério do Meio Ambiente, Brasília, p. 285-299.

Companhia de Pesquisas de Recursos Minerais - CPRM. 2006. Mapa geológico do Estado do Rio Grande do Sul - 1:750.000. Disponível em: http://www.cprm.gov.br/publique/media/mapa_rio_grande_sul. pdf. Acessado em 05.06.2015.

Centro de Sensoriamento Remoto - CSR/IBAMA. 2011. Monitoramento do desmatamento nos biomas brasileiros por satélite. Acordo de cooperação técnica MMA/IBAMA. Monitoramento bioma Pampa 2008 e 2009. Disponível em: http://mma.gov.br/estruturas/ sbf_chm_rbbio/_arquivos/relatrio_tcnico_monitoramento pampa_2008_2009_72.pd. Acessado em 18.01.2016.

Dresseno, A.L.P. \& Overbeck, G.E. 2013. Structure and composition of a grassland relict within an urban matrix: potential and challenges for conservation. Iheringia. Série Botânica 68:59-71.

Ferreira, P.M.A., Müller, S.C., Boldrini, I.I. \& Eggers, L. 2010. Floristic and vegetation structure of a granitic grassland in Southern Brazil. Revista Brasileira de Botânica 33:21-36.

Filgueiras, T.S., Nogueira, P.E., Brochado A.L. \& Guala II, G.F. 1994. Caminhamento - um método expedito para levantamentos florísticos qualitativos. Cadernos de Geociências 12:39-43.

Frizzo, T.C.E. \& Porto, M.L. 2004. Zoneamento da vegetação e sua relação com a ocorrência de estruturas mineralizadas na Mina Volta Grande, Lavras do Sul, RS, Brasil. Iheringia. Série Botânica 59(1):5-12.

Gibson, D.J. 2009. Grasses and grassland ecology. Oxford, New York. $305 \mathrm{p}$.

Goffermann, M., Viero, A.P. \& Da Silva, E.B. 2015. Caracterização hidrogeológica e hidroquímica das águas subterrâneas da região de São Gabriel, RS. Pesquisas em Geociências 42(3):239-261.

Hasenack, H., Weber, E., Boldrini, I.I. \& Trevisan, R. 2010. Mapa de sistemas ecológicos da ecorregião das savanas uruguaias em escala 1:500.000 ou superior e relatório técnico descrevendo insumos utilizados e metodologia de elaboração o mapa de sistemas ecológicos. The Nature Conservancy, Relatório Técnico. 18p.

Instituto Brasileiro de Geografia e Estatística - IBGE. 2003. Mapa geomorfológico do município de São Gabriel (folha SH. 21-ZB). Disponível em: ftp://geoftp.ibge.gov.br/mapas_tematicos/ geomorfologia/cartas_escala_250mil/sh21zb_geom.pdf. Acessado em 11.04.2015.

. 2004. Mapa de Biomas do Brasil. Disponível em: http://www. ibge.gov.br/home/presidencia/noticias/21052004biomashtml.shtm. Acessado em 11.04.2015.

Internation Plant Names Índex - IPNI. 2015. Disponível em: http://www. ipni.org $>$. Acessado em 11.04.2015.

Keddy, P.A. 1992. Assembly and response rules: two goals for predictive communityecology. Journal of Vegetation Science 3:157-164.

Lindman, C.A.M. \& Ferri, M.G. 1974. A vegetação no Rio Grande do Sul (Brasil austral). Ed. Itatiaia, Belo Horizonte. 377p.

Lista de espécies da flora do Brasil. 2015. Disponível em: http:// floradobrasil.jbrj.gov.br/jabot/listaBrasil/PrincipalUC/PrincipalUC. do;jsessionid=80FDEDF1F261720BE931307BAD26A59F. Acessado em 11.04.2015.

Modernel, P., Rossing, W.A.H., Coorbels, M., Dogliotti, S., Picasso, V. \& Tittonell, P. 2016. Land use change and ecosystem servisse provision in Pampas and Campos grasslands of southern South America. Environmental Research Lettters 11:1-21.

Mourelle, D. 2011. Relación polen-vegetación actual en Uruguay. Dissertação 61 f. Universidad de la Republica Uruguay, Montevideo.

Müeller-Dombois, D. \& Ellenberg, H. 1974. Aims and methods of vegetation ecology. John Wiley, New York. 547p.

Müller, N.L. 1962. A região de São Gabriel. Associação dos geógrafos brasileiros, São Paulo. 77p.

Overbeck, G.E., Müller, S.C., Fidelis, A., Pfadenhauer, J., Pillar, V.P., Blanco, C.C., Boldrini, I.I., Both, R. \& Forneck, E.D. 2007. Brazil's neglected biome: The South Brazilian Campos. Perspectives in Plant Ecology. Evolution and Systematics 9:101-116.
Overbeck, G.E., Hermann, J.M., Andrade, B.O., Boldrini, I.I., Kiehl, K., Kirmer, A., Koch, C., Kollmann, J. Meyer, S.T., Müller, S.C., Nabiger, C., Pilger, G.E., Trindade, J.P.P., Vélez-Martim, E., Walker, E.A., Zimmermann, D.G. \& Pillar, V.P. 2013. Restoration Ecology in Brazil - Time to Step Out of the Forest. Natureza \& Conservação 11(1):92-95.

Overbeck, G.E., Müller, S.C., Pillar, V.D. \& Pfadenhauer, J. 2006. Floristic composition, environmental variation and species distribution patterns in burned grassland in Southern Brazil. Brazilian Journal of Biology 66(4):1073-1090.

Overbeck, G.E., Vélez-Martim, E., Scarano, F.R. Lewinsohn, T.M., Fonseca, C.R., Meyer, S.T., Muller, S.C., Ceotto, P. Dadalt, L., Durigan, G., Ganade, G., Gossner, M.M., Guadagnin, D.L., Lorenzen, K., Jacobi, C.M., Weisser, W.W. \& Pillar, V.P. 2015. Conservation in Brazil needs to include non-forest ecosystems. Diversity and Distributions 1-6.

Peel, M.C., Finlayson, B.L. \& Mcmahon, T.A. 2007. Updated world map of the Köppen-Geiger climate classification. Hydrology and Earth Sciences 11:1633-1644.

Pillar, V.P. 1999. The bootstrapped ordination reexamined. Journal of Vegetation Science 10:895-902.

.2006. MULTIV: Multivariate Exploratory Analysis, Randomization Testing and Bootstrap Resampling. User's Guide v. 2.4. Universidade Federal do Rio Grande do Sul, Porto Alegre. 51p.

Pillar, V.P., Andrade, B.O. \& Dadalt, L. 2015. Serviços ecossistêmicos. In Os campos do Sul. (V.P. Pillar \& O. Lange, eds). Rede campos sulinos, Porto Alegre, p. 115-121.

Porto, M.L. 2002. Os campos sulinos: sustentabilidade e manejo. Ciência \& Ambiente 24:119-138.

Rambo, B. 1956. A fisionomia do Rio Grande do Sul. Livraria Selbach, Porto Alegre. 456p.

Reinert, D.J., Reichert, J.M., Dalmolin, R.S.D., Azevedo, A.C. \& Pedron, F.A. 2007. Principais solos da Depressão Central e Campanha do Rio Grande do Sul. Gráfica Universitária da Universidade de Santa Maria, Santa Maria. 47p.

Rolim, R.G., Setubal, R.B., Casagrande, A., Rivas, M.I.E., De Nardin, J.A., Proença, M.L. Sandri, S.M., Bonilha, C.L. \& Boldrini, I.I. 2014. Composição e estrutura de vegetação campestre em áreas com orientação norte e sul no Jardim Botânico de Porto Alegre, RS, Brasil. Iheringia. Série Botânica 69(2):433-449.

Setubal, R.B. \& Boldrini, I.I. 2010. Floristic and characterization of grassland vegetation at a granitic hill in Southern Brazil. Revista Brasileira de Biociências 8:85-111.

2012. Phytosociology and natural subtropical grassland communities in a granitic hill in southern Brazil. Rodriguésia 63:513-524.

Sippel, C. 2003. Unidades da paisagem e suas relações com características dos solos na área da mineração Volta Grande, Lavras do Sul, RS Uma visão em diferentes escalas. Dissertação 192f., Universidade Federal do Rio Grande do Sul, Porto Alegre.

Soriano, A. 1991. Río de la Plata grasslands. In Ecosystems of the world 8A. Natural Grasslands (R.T. Coupland. ed.). Elsevier, New York, p. 367-369.

Streck, E.V., Kämpf, N., Dalmolin, R.S.D., Klamt, E., Nascimento, P.C. do, Schneider, P., Giasson, E. \& Pinto, L.F.S. 2008. Solos do Rio Grande do Sul. Emater, RS, Porto Alegre. 222p.

Tonello, M.S. \& Prieto, A.R. 2008. Modern vegetation-pollen-climate relationships for the Pampa grasslands of Argentina. Journal of Biogeography 35:926-938.

Vedana, L.A. \& Philipp, R.P. 2016. Análise petrográfica e proveniência dos metassedimentos do Complexo Pontas do Salso, Terreno São Gabriel, Cinturão Dom Feliciano, RS. Revista Pesquisas em Geociências 43(3):229-248.

Wrege, M.S., Steinmentz, S., Reisser-Júnior, C. \& Almeida, I.R. 2011. Atlas climático da região Sul do Brasil, Estados do Paraná, Santa Catarina e Rio Grande do Sul. Embrapa, Pelotas. 333p.

Zocche, J.J. 2002. Comunidades vegetais de savana sobre estruturas mineralizadas de cobre, na Mina Volta Grande, Lavras do Sul, RS. Tese 248 f., Universidade Federal do Rio Grande do Sul, Porto Alegre. 